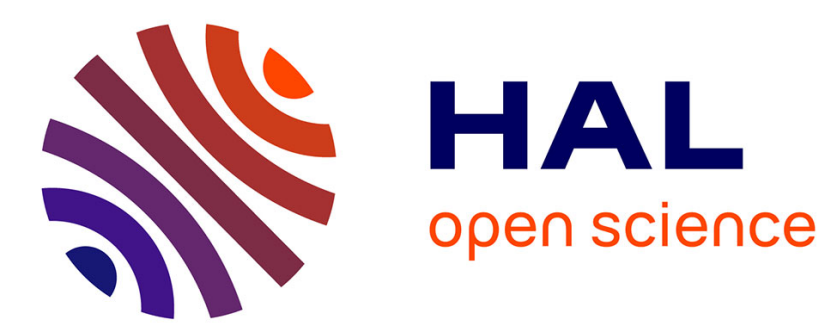

\title{
A Eulerian Method for Capturing Caustics
}

Jean-David Benamou, Ian Solliec

\section{To cite this version:}

Jean-David Benamou, Ian Solliec. A Eulerian Method for Capturing Caustics. [Research Report] RR-3786, INRIA. 1999. inria-00072873

\section{HAL Id: inria-00072873 https://hal.inria.fr/inria-00072873}

Submitted on 24 May 2006

HAL is a multi-disciplinary open access archive for the deposit and dissemination of scientific research documents, whether they are published or not. The documents may come from teaching and research institutions in France or abroad, or from public or private research centers.
L'archive ouverte pluridisciplinaire HAL, est destinée au dépôt et à la diffusion de documents scientifiques de niveau recherche, publiés ou non, émanant des établissements d'enseignement et de recherche français ou étrangers, des laboratoires publics ou privés. 
INSTITUT NATIONAL DE RECHERCHE EN INFORMATIQUE ET EN AUTOMATIQUE

\title{
A Eulerian Method for Capturing Caustics
}

\author{
Jean-David Benamou , Ian Solliec
}

\section{$\mathbf{N}^{\circ} 3786$}

Octobre 1999

THÈME 4 



\title{
A Eulerian Method for Capturing Caustics
}

\author{
Jean-David Benamou* , Ian Solliec ${ }^{\dagger}$ \\ Thème 4 - Simulation et optimisation \\ de systèmes complexes \\ Projet Ondes \\ Rapport de recherche $\mathrm{n}^{\circ} 3786$ - Octobre 1999 - pages
}

\begin{abstract}
A robust numerical method for the localization of caustics is proposed for general Hamiltonians. It is based on the direct resolution of a system of partial differential equations obtained through a local change of the time variable in the Hamilton-Jacobi equation and complemented by a set of transport equation. Numerical results (1 to $3-\mathrm{D})$ are presented.
\end{abstract}

Key-words: Hamilton-Jacobi, Free Boundary, Caustic, Hamiltonian System, Ray Tracing, Viscosity Solution, Upwind Scheme, Adaptive Grid Refinement.

* INRIA, Domaine de Voluceau, B.P.105 78153 Le Chesnay cedex, France.

$\dagger$ INRIA and CEA. 


\section{Une méthode Eulérienne de capture de caustiques}

Résumé : Une méthode numérique robuste de localisation de caustiques est proposé pour des Hamiltoniens généraux. Elle est basée sur la résolution directe d'un système d'équations aux dérivées partielles obtenu à l'aide d'un changement de variables local en temps dans l'équation d'Hamilton-Jacobi et complémenté d'un système d'équations de transport. Des résultats numériques (1 à 3-D) sont présentés.

Mots-clé : Hamilton-Jacobi, Frontière Libre, Caustique, Système Hamiltonien, Lancer de Rayons, Solution de Viscosité, Schéma Décentrés, Raffinement Adaptatif de Maillage. 


\section{Table of Contents}

1 Introduction $\quad 3$

2 Basic tools $\quad 4$

2.1 The Lagrangian method $\ldots \ldots \ldots \ldots \ldots \ldots \ldots \ldots \ldots$

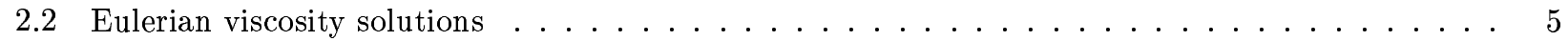

3 Eulerian pathologies $\quad 6$

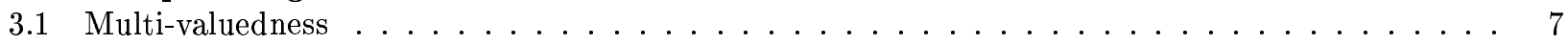

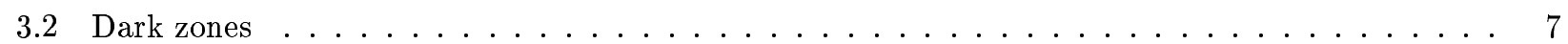

3.3 Combination of the two $\ldots \ldots \ldots \ldots \ldots \ldots \ldots$

4 Eulerian caustic capturing $\quad 8$

4.1 The Lagrangian version $\ldots \ldots \ldots \ldots \ldots \ldots \ldots$

4.2 From Lagrangian to Eulerian $\ldots \ldots \ldots \ldots \ldots \ldots$

4.3 More on Eulerian/Lagrangian correspondence . . . . . . . . . . . . . . . . . . 11

4.4 1-D Numerical results . . . . . . . . . . . . . . . . . . . . . . . . . . . . . 12

4.5 Remark on the choice of $\tilde{s}(s) \ldots \ldots \ldots \ldots \ldots \ldots \ldots \ldots \ldots$

$5 \quad$ 2-3 Dimensional results $\quad 12$

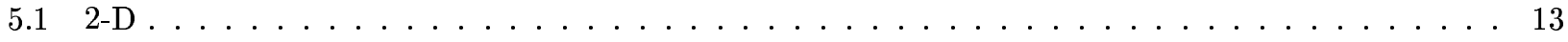

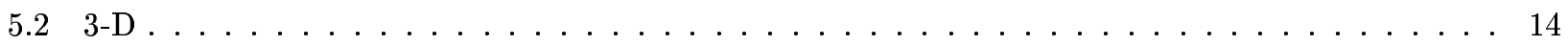

6 Conclusion

\section{Introduction}

Caustics are commonly observed as an optical phenomena and the easiest and intuitive definition of such an object relies on light rays : a caustic is the envelope of a family of rays. This is also a place where rays concentrate and the pattern of a caustic can easily be distinguished as light intensity increases dramatically there ("kaustikos" means burning in ancient Greek). This phenomena occurs when rays are bent either by diffraction or by the variation of the optical properties of the medium; or simply when the source of the light is such that the rays focus (remember the parabolic mirrors of Archimedes to set on fire the Roman war fleet using the rays of the sun).

Of course the actual perception we have of this phenomena is not really rays (no one has ever observed a single light ray). It would be more accurate to speak of a continuum or a field of rays. Rays are simply a convenient and natural way of modeling and understanding the underlying phenomena. In this paper, numerical methods based on rays are called Lagrangian because of the analogy between rays and particle trajectories in fluid mechanics : various physical quantities are computed but only along characteristic curves which themselves depends on the physics. In this framework, determining caustics points on rays is easy. The main draw back of the Lagrangian method is the difficulty to maintain a uniform resolution in space even when increasing the number of computed rays. Caustic curves and surfaces (in 3-D) are then represented by a collection of possibly sparse points belonging to different rays. The continuous vision we have of this optical phenomena is therefore difficult to achieve numerically in the Lagrangian framework (a possible fix is to use sophisticated representation/interpolation techniques as in [26] [16]).

An alternate modeling possibility, in some sense closer to our visual perception, is to use functions defined on the continuous medium and which also describe the propagation of light. They are generally called phase (or travel-time or eikonal) and amplitude (or intensity) and they solve two partial differential equations : respectively a Hamilton-Jacobi equation and a transport equation. Numerical methods based on this approach are called Eulerian : the discretization of the support of the solution is fixed a priori and in particular does not depend on the rays. The space resolution of the numerical method is therefore easily maintained and the accuracy of the approximation depends on this resolution. One issue of geometric optics is to formalize the relationship between rays, phase and amplitude. In particular, this theory predicts an infinite amplitude at caustics or equivalently that the local dilatation rate of the field of rays is 0 . The present piece of work shows 
that it is possible, but not straightforward, to determine caustics in the Eulerian framework.

It is well known that Lagrangian solutions, which are the bicharacteristics of a given Hamiltonian system, match the Eulerian solutions of the corresponding Hamilton-Jacobi equation as long as they are "classical" [14] [27]. This means that rays are not allowed to cross or equivalently that the phase transported by the rays remains a Eulerian function of space : it is singled valued and defined everywhere. This assumption is right at the heart of the problem and we can identify two distinct sources of trouble.

First, while the Lagrangian method naturally sweeps several time the same space location producing a multivalued solution, the Eulerian method only computes a single-valued solution. It is known that in multi-valued regions, the so called viscosity solution (the output of stable upwind schemes) only yields the minimum phase pointwise [25] [5]. Quite a number of attempts at producing Eulerian or mixed Eulerian/Lagrangian methods to solve the multi-valued problem can be found in [22] [11] [4] [1] [18] [8] [12].

The second issue is the problem of determining caustics as free boundaries for the Eulerian approach. The caustic location indeed depends on the solution itself which is only defined on one side of the caustic envelope itself.

In [5], where a generic algorithm for the splitting of the multi-valued solution into several single-valued Eulerian solutions is presented, a heuristic method was proposed to locate caustics. This work is probably the first to address this problem as caustics are generically never associated to the minimum phase and cannot be observed in plain viscosity solutions.

In this paper, we focus on caustic capturing and we explain how the two fundamental difficulties mentioned above combine to make the problem of Eulerian caustic capturing difficult. Then, we propose a robust algorithm to do so.

Section 2 recalls the bases of Lagrangian and Eulerian approach. Section 3 discusses the different problems we face when using Eulerian methods. Section 4 presents the Eulerian caustic capturing algorithm. Section 2 to 4 are written for a general Hamiltonian and illustrated by one dimensional numerical simulations. Section 5 presents 2-D and 3-D numerical caustic capturing for a problem modeling laser beam propagation in a plasma.

\section{Basic tools}

\subsection{The Lagrangian method}

A Hamiltonian function $H(t, y, p)$ is given, defined on $\mathbb{R}_{t}^{+} \times \mathbb{R}_{y}^{d} \times \mathbb{R}_{p}^{d}, d$ is the space dimension of the problem, $\mathbb{R}_{t}^{+} \times \mathbb{R}_{y}^{d}$ is the time-space configuration in which rays can evolve and $\mathbb{R}_{y}^{d} \times \mathbb{R}_{p}^{d}$ is called the phase space. The Hamiltonian is assumed to be continuous up to its second derivatives and convex and coercive in its last variable $p$.

The Lagrangian method then consists in solving the Hamiltonian system formed by the following set of ordinary differential equations (ODEs) [27] [2] :

$$
\left\{\begin{array}{l}
\dot{y}\left(s, x^{0}\right)=H_{p}\left(s, y\left(s, x^{0}\right), p\left(s, x^{0}\right)\right), y\left(0, x^{0}\right)=x^{0}, \\
\dot{p}\left(s, x^{0}\right)=-H_{y}\left(s, y\left(s, x^{0}\right), p\left(s, x^{0}\right)\right), p\left(0, x^{0}\right)=\phi_{x^{0}}^{0}\left(x^{0}\right), \\
\dot{\varphi}\left(s, x^{0}\right)=p\left(s, x^{0}\right) \cdot H_{p}\left(s, y\left(s, x^{0}\right), p\left(s, x^{0}\right)\right)-\ldots \\
H\left(s, y\left(s, x^{0}\right), p\left(s, x^{0}\right)\right), \varphi\left(0, x^{0}\right)=\phi^{0}\left(x^{0}\right) .
\end{array}\right.
$$

The dot stands for time derivation $(\dot{.})=\frac{d(.)}{d s}, g_{x_{i}}\left(x_{1}, x_{2}\right)$ and $g_{x_{i} x_{j}}\left(x_{1}, x_{2}\right)$ respectively denote the gradient and the Hessian of $g$ with respect to $x_{i}$ and $\left(x_{i}, x_{j}\right), \phi^{0}$ is the initial phase and also appears in the initial condition for $p$. For each $x^{0} \in \mathbb{R}_{y}^{d}$ (or a subset of $\mathbb{R}_{y}^{d}$ ), the system generates "bicharacteristics strips" $\left(y\left(s, x^{0}\right), p\left(s, x^{0}\right)\right.$ ) lying in phase-space with smooth dependence on $s$ and $x^{0}$. The projections of the strips onto $\mathbb{R}_{y}: y\left(s, x^{0}\right)$, are called the rays. Each ray is therefore "labeled" by its initial position $x_{0}$. The phase $\varphi\left(s, x^{0}\right)$ is transported by the corresponding ray $y\left(s, x^{0}\right)$ and, when rays are crossing, is a multi-valued function of the configuration space $\mathbb{R}_{t}^{+} \times \mathbb{R}_{y}^{d}$

It happens in particular at caustics which are the points on the rays where an infinitesimal tube of neighboring rays collapses. Mathematically, a ray $y\left(s, x^{0}\right)$ encounters a caustic point when the determinant (denoted |.|) of 
the Jacobian matrix of $y$ with respect to $x^{0}$ :

$$
\alpha\left(s, x^{0}\right)=\left|\frac{\partial y\left(s, x^{0}\right)}{\partial x^{0}}\right|
$$

is zero. The quantity $\alpha$ is sometimes called "geometrical spreading" as it provides a local measure of the geometrical convergence or divergence of the rays.

The Lagrangian method therefore needs to evaluate $\alpha\left(s, x^{0}\right)$ along the rays to locate caustic points. The computation of $\frac{\partial y\left(s, x^{0}\right)}{\partial x^{0}}$ is performed using a set of additional ODEs obtained via a linearization of the system (1) with respect to $x^{0}$ :

$$
\left\{\begin{array}{l}
\left(\begin{array}{l}
\frac{\dot{\partial} y}{\partial x^{0}}\left(s, x^{0}\right) \\
\frac{\dot{\partial} p}{\partial x^{0}}\left(s, x^{0}\right)
\end{array}\right)=A\left(s, y\left(s, x^{0}\right), p\left(s, x^{0}\right)\right) \cdot\left(\begin{array}{c}
\frac{\partial y}{\partial x^{0}}\left(s, x^{0}\right) \\
\frac{\partial p}{\partial x^{0}}\left(s, x^{0}\right)
\end{array}\right), \\
\left(\begin{array}{l}
\frac{\partial y}{\partial x^{0}}\left(0, x^{0}\right) \\
\frac{\partial p}{\partial x^{0}}\left(0, x^{0}\right)
\end{array}\right)=\left(\begin{array}{l}
I d_{d \times d} \\
\frac{\partial^{2} \phi^{0}}{\partial x^{02}}\left(x^{0}\right)
\end{array}\right)
\end{array}\right.
$$

where

$$
A(t, y, p)=\left(\begin{array}{cc}
H_{p y}(s, y, p) & H_{p p}(s, y, p) \\
-H_{y y}(s, y, p) & -H_{y p}(s, y, p)
\end{array}\right) .
$$

This is a set of $2 d^{2}$ ODEs but as it is written each unknown of system (3) is a $d \times d$ matrix and the matrix vector product must be understood in the correct algebra.

Lagrangian caustic localization therefore consists in solving (1-3) and evaluate $\alpha\left(s, x^{0}\right)$ along a ray using (2). When it vanishes or more precisely changes its sign, the ray has passed a caustic point.

\subsection{Eulerian viscosity solutions}

The Eulerian method is based on the alternate formulation of the problem as an Hamilton-Jacobi equation

$$
\left\{\begin{array}{l}
\frac{\partial \phi}{\partial t}(t, x)+H\left(t, x, \phi_{x}(t, x)\right)=0, \text { for }(t, x) \in \mathbb{R}_{t}^{+} \times \mathbb{R}_{y}^{d} \\
\phi(0, x)=\phi^{0}(x), \text { for } x \in \mathbb{R}_{y}^{d},
\end{array}\right.
$$

When the solution is classical, typically $\phi^{0}$ is $C^{1}$ and $\alpha$ does not vanish, a classical result of the calculus of variation [14] [27] proves that the Eulerian phase $\phi(t, x)$ evaluated at the Lagrangian coordinates specified by the rays matches the Lagrangian phase and its gradient the $p$ components of the bicharacteristics :

$$
\left\{\begin{array}{l}
\phi\left(s, y\left(s, x^{0}\right)\right)=\varphi\left(s, x^{0}\right) \\
\phi_{x}\left(s, y\left(s, x^{0}\right)\right)=p\left(s, x^{0}\right)
\end{array}\right.
$$

We will come back to this correspondence and prove it as a particular case of our new Eulerian formulation in section 4.3.

When the conditions for a classical solution are not satisfied, there is still a notion of global weak solution for equation (5) called "viscosity solution" [9] [3]. Viscosity solutions are the correct object to consider here because any "reasonable" numerical scheme converges to this class of solution [21] [10]. These schemes are generally called upwind because they discretize space derivatives on the opposite side to the direction of the rays (would rays be traced).

A link can still be made between Lagrangian and Eulerian solution using the theory of optimal control (see [5]). The viscosity solution can be characterized as the value function of the following optimization problem

$\mathrm{RR} \mathrm{n}^{\circ} 3786$

$$
\phi(t, x)=\inf _{\substack{\left\{x^{0} \in \mathbb{R}_{y}, y(.) \in W^{1,+\infty}(\mathbb{R}) ; \\ y(0)=x^{0}, y(t)=x\right\}}} \int_{0}^{t} L(s, y(s), \dot{y}(s)) d s+\phi^{0}\left(x^{0}\right),
$$


where the minimization is performed with respect to the admissible curves $y($.$) and their initial point x^{0}$. The Lagrangian function $L(t, x, v)=\sup _{p \in \mathbb{R}_{p}}\{p \cdot v-H(t, x, p)\}$ is the Legendre transform of $H$ with respect to $p$.

When only one ray matches the end point condition and has no caustic point, then the solution is "classical" and the value function of problem (7) is exactly the integral of the phase ODE in (1).

If more than one ray reaches the time space point (still with no caustic points) $(t, x)$ and if we denote $\left(x_{k}^{0}\right)_{k=1 . . n}$ the $n$ initial points of these curves, then the viscosity solution $\phi$ selects the absolute minimum of the associated phases :

$$
\phi(t, x)=\min _{k=1 . . n} \varphi\left(t, x_{k}^{0}\right) .
$$

If no ray reaches this point, the viscosity solution implicitly generates "non-classical" rays to fill this empty space. It means that the optimal curves will satisfy the Hamiltonian system (1) with different initial conditions on the $p$ component. We discuss this phenomena in the next section as well as the behavior of the viscosity solution in the presence of caustics.

When the configuration space is bounded, the optimal curves may only satisfy the ray equations (1) piecewise (they can be reflected or diffracted) or they can creep along boundaries. This will not be the case in this paper (see [15] for an investigation of this question).

Of course (5) alone only provides information on the phase. As in the Lagrangian method we will need additional equations to determine caustics. We therefore introduce a new Eulerian vector valued variable $\delta(t, x)$ configured in time-space $(t, x) \in \mathbb{R}_{t}^{+} \times \mathbb{R}_{y}^{d}$ and defined by $\left(.^{T}\right.$ is the transpose of .)

$$
\delta\left(s, y\left(s, x^{0}\right)\right)=\left(\frac{\partial y\left(s, x^{0}\right)}{\partial x^{0}}, \frac{\partial p\left(s, x^{0}\right)}{\partial x^{0}}\right)^{T} .
$$

As for the Hamilton-Jacobi (see section 4.3), each component of $\delta(t, x), \delta_{i}(t, x)$ can be shown to satisfy the transport equation

$$
\left\{\begin{array}{l}
\frac{\partial \delta_{i}}{\partial t}(t, x)+H_{p}\left(t, x, \phi_{x}\right) \cdot \delta_{i, x}(t, x)=A\left(t, x, \phi_{x}(x)\right) \cdot \delta_{i}(t, x), i=1,2 \\
\delta(0, x)=\left(1_{\mathbb{R}^{d}}, \frac{\partial^{2} \phi^{0}}{\partial x^{02}}\left(x^{0}\right)\right)^{T}, \text { for } x \in \mathbb{R}^{d} .
\end{array}\right.
$$

As in (3) the unknowns $\delta_{i}$ are $d \times d$ matrix and we here have a matrix equations where the differential operators must be applied component-wise and the - indicates the proper inner multiplication. The solution of this system, when coupled to (5), gives the necessary information to compute a Eulerian counterpart for $\alpha$ denoted $\beta(t, x)$ :

$$
\beta\left(s, y\left(s, x^{0}\right)\right)=\alpha\left(s, x^{0}\right) .
$$

Finally $\beta$ can simply be expressed only in terms of Eulerian variables (using (2) (9)):

$$
\beta(t, x)=\left|\delta_{1}(t, x)\right|
$$

Alternate methods for the computation of $\beta$ can be found in [13] [7] [23].

The Eulerian method should therefore be able to determine caustic points using $\beta$. The situation is however not so simple because the restrictions imposed by the properties of the viscosity solution (i.e. (7) and (8)) do not allow direct access to these points. This is the subject of the next section and the Eulerian way to caustics is presented in section 4 .

\section{Eulerian pathologies}

These phenomena are generically linked to the occurrences of caustics. In 2-D (including the time dimension), fold or cuspidal caustics are generic for general Hamilton-Jacobi equations. We send back to [5] and the references therein for more on this topic. In higher dimension there may be additional type of caustics but the pathologies discussed in this section are general. 
We concentrate on a particular Hamiltonian for a problem in 1-D space dimension arising from 2-D geometric optics (see [25] or [5])

$$
H(t, x, p)=-\sqrt{n^{2}(t, x)-p^{2}},(t, x, p) \in \mathbb{R}_{t}^{+} \times \mathbb{R}_{y} \times \mathbb{R}_{p},
$$

where $n(t, x)$ is a positive smooth index of refraction.

\subsection{Multi-valuedness}

With the particular choice (13), multi-valuedness may occur either because of the variation $n$ (as for a lens) or even when $n$ is constant but the initial phase $\phi^{0}$ is such that rays will focus.

Figure 1 shows rays corresponding to

$$
\left\{\begin{array}{l}
n \equiv 3, \\
\phi^{0}(x)=-\frac{\sqrt{1+16 *(x-0.5)^{2}}}{2}
\end{array}\right.
$$

This particular choice leads to converging initial direction for the rays (given by $\left.\dot{y}\left(0, x^{0}\right)=H_{p}\left(0, x^{0}, \phi_{x^{0}}^{0}\left(x^{0}\right)\right)\right)$ ). Rays focus, and form an envelope called a cuspidal caustic. The system (1-3) is solved using a 4th order RungeKutta method for rays equally spaced on $t=0$. When $\alpha$ changes its sign (here from positive to negative) the rays change from plain line to dashed line. The caustic points are marked with red stars. Inside the caustic envelope rays are crossing. More precisely, if we could visualize the rays associated to all labels $x^{0}$ on the real axis, each point would be passed by three rays and the associated phase is triple-valued.

On figure 2, we superimposed the level curves of the viscosity solution (the color map correspond to the value of the phase). It is computed on a Cartesian grid using a second order ENO Godunov scheme in space and an adaptive third order Runge-Kutta in time [17] [24]. We use out-going boundary conditions, so we implicitly assume that rays are flowing outward. It is, of course, not necessarily true but does not infer with the solution in the caustic zone. In this case it produces a "rarefaction" effect near the boundaries. We come back on this phenomena in the next section. The usual observation is that rays and "fronts" (the color level curves) are orthogonal. This is simply because the direction of the rays is given by $\phi_{x}$ when $H_{p}$ is collinear to $p$ (see (6)). It is therefore possible to keep tract visually of the Lagrangian to Eulerian correspondence. In the multi-valued zone, the viscosity solution picks up only one of the coexisting rays according to rule (8). It produces a curve of singularities in the gradient of $\phi$ called a kink (or shock) when the solution "jumps" from left going rays to right going rays.

The important remark is that the kink occurs on the rays before they reach their caustic points with the notable exception of the cusp which which is a kink and a caustic point at the same time (and plays a fundamental role in the algorithm proposed in [5]).

So, even when solving (10) coupled to (5) and evaluating $\beta$, the viscosity solution misses the caustics. This can be seen on figure 3 where a color map of $\beta$ is displayed. The kink can be observed and $\beta$ take a zero value only at the cusp and the rest of the caustic cannot be seen as it "belongs" to later "branches" of the multi-valued solution.

\subsection{Dark zones}

There can be zones with no rays at all for several reason (we already observed this phenomena in the previous example). We present the simplest case here but the next section will show that it is a general rule with caustics.

We keep the same constant index of refraction but now chose

$$
\phi^{0}(x)=\frac{3 *|x-0.5|}{\sqrt{2}}
$$

which has a singularity at $x=0.5$.

On each side of 0.5 it is possible to trace classical rays. Several of them are plotted in figure 4 and of course there are no caustic in this case.

$\mathrm{RR} \mathrm{n}^{\circ} 3786$ 
The interesting phenomena occurs when we proceed to compute the viscosity solution. The level line of the phase are again superimposed on the rays in figure 5. Of course the viscosity solution does not know that the classical rays do not reach the middle region (the dark zone) and fills it according to rule (7). The solution actually behaves as a propagating front which initially has a corner at $t=0, x=0.5$. The corner generates a diffracted circular front which fills the dark zone. The viscosity solution implicitly generates diffracted rays (imagine a fan of rays in the dark zone). We refer to [15] for a mathematical investigation of the link between diffraction and viscosity solutions.

It must be said that these two effects exactly correspond to shock and rarefaction waves for scalar Hyperbolic conservation laws like Burgers equation (just differentiate the Hamilton-Jacobi equation in space).

\subsection{Combination of the two}

Really bad things happen when these two phenomena (described in section 3.1 and 3.2) combine in the presence of caustics.

We are now considering a variable index of refraction made of a constant part in which the rays are straight lines and a vertical layer which bends the rays in the negative $x$ direction

$$
\left\{\begin{array}{c}
n(x)=1, \text { for } x \leq 0.5 \\
n(x)=1-(x-0.5)^{3}, \text { for } x>0.5
\end{array}\right.
$$

The initial phase prescribes an incoming oblique plane wave

$$
\phi^{0}(x)=\frac{x}{\sqrt{2}},
$$

and we add a compatible boundary condition at $x=0$ which also generates incoming rays to avoid rarefaction effects on the left side :

$$
\phi_{x}(t, 0)=\frac{1}{\sqrt{2}}, \forall t .
$$

It generates a stationary solution when the contribution of the rays associated to the initial Cauchy condition have disappeared. The rays are presented in figure 6 and we observe a folded caustic. It is as if we only consider half of a cuspidal caustic. Dashed line again represent rays after they passed a caustic point (red stars). Notice the dark zone on the right of the caustic (which is by definition the envelope of the rays).

The viscosity solution in figure 7 exhibits a kink lying on the left of the caustic. It is produced by the conflicting classical rays and diffracted rays (not represented) which implicitly correspond to the viscosity solution in the dark zone and are bent back in the illuminated zone. The post caustic rays (dashed lines) are not represented by the viscosity solution as they produce later phases. Classical and diffracted rays actually generate a new cuspidal caustic and classical rays are implicitly "blocked" by a kink before reaching the caustic by the diffracted rays. It can be seen on the geometrical spreading (figure 8 ) where $\beta$ is really discontinuous along the kink and only takes a relevant zero value at the tip of the caustic.

\section{Eulerian caustic capturing}

Section 3.3 diagnoses the "pollution" effect which a priori prevents the viscosity solution to capture caustics. We insists again on the idea that this is a generic pathology which may occur for any Hamilton-Jacobi equation in any dimension. More or less successful heuristic attempts at fixing locally the Eulerian upwind scheme are proposed in [5] [6] [19].

We present in this section a general approach valid for any dimension and illustrate it on the test case of section 3.3. We first present it as a modification of the Lagrangian method (even though there is nothing really to fix in this framework except the space resolution of the method) and then explain how to convert this idea to the Eulerian framework. 


\subsection{The Lagrangian version}

The idea of the fix is easier to present with rays. As explained in section 3, the Eulerian viscosity solution follows classical rays as long they exist and yield minimum phase. Trouble starts as soon as the tip of a caustic is reached. Rays then leave an empty zone and the viscosity solution sees the effect of new polluting diffracted rays.

The idea of the method is to modify (1-3) such that rays will reach the caustic but only asymptotically in time. A change of variable in time depending on $\alpha$ is used to that effect. More precisely, we define a new time $\tilde{s}$ along each ray $y\left(s, x^{0}\right)$ given by

$$
\tilde{s}\left(s, x^{0}\right)=\int_{0}^{s} \frac{1}{\alpha\left(\sigma, x^{0}\right)} d \sigma .
$$

For convenience, we reset our equations in a general framework, let

$$
U\left(s, x^{0}\right)=\left(y\left(s, x^{0}\right), p\left(s, x^{0}\right), \varphi\left(s, x^{0}\right), \frac{\partial y}{\partial x^{0}}\left(s, x^{0}\right) \frac{\partial p}{\partial x^{0}}\left(s, x^{0}\right)\right)^{T},
$$

then, the Lagrangian system (1-3) can be compactly written in a general form ( $F, G$ and $U^{0}$ are defined using $(1-3)$ and (2)) :

$$
\dot{U}\left(s, x^{0}\right)=F\left(s, U\left(s, x^{0}\right)\right), U\left(0, x^{0}\right)=U^{0}
$$

and $\alpha\left(s, x^{0}\right)$ can be written as a function of $U$ :

$$
\alpha\left(s, x^{0}\right)=G\left(U\left(s, x^{0}\right)\right) .
$$

The new rays are obtained simply be changing from time $s$ to time $\tilde{s}\left(s, x^{0}\right)(19)$. Let us set

$$
\tilde{U}\left(\tilde{s}\left(s, x^{0}\right), x^{0}\right)=U\left(s, x^{0}\right),
$$

which naturally satisfies the modified system

$$
\left\{\begin{array}{l}
\dot{\tilde{U}}\left(\tilde{s}, x^{0}\right)=\tilde{\alpha}\left(\tilde{s}, x^{0}\right) F\left(\tilde{s}, \tilde{U}\left(\tilde{s}, x^{0}\right)\right), \tilde{U}\left(0, x^{0}\right)=U^{0} \\
\tilde{\alpha}\left(\tilde{s}, x^{0}\right)=G\left(\tilde{U}\left(\tilde{s}, x^{0}\right) .\right.
\end{array}\right.
$$

Of course, the dot here stands for the new time derivation $()=.\frac{d(.)}{d \tilde{s}}$. It is easy to check that $\tilde{\alpha}\left(\tilde{s}\left(s, x^{0}\right), x^{0}\right)=$ $\alpha\left(s, x^{0}\right)$ and therefore the system (24) will either be stationary when it reaches the caustic or only reach it asymptotically in time. Either options depend on the convergence or divergence of the integral (19) as the ray approaches the caustic.

In our case, $\alpha\left(s, x^{0}\right)$ is a decreasing function of time $s$ and is zero, say at time $s^{*}$, when the ray passes a caustic point $y\left(s^{*}, x^{0}\right)$. When $\dot{\alpha}\left(s^{*}, x^{0}\right)$ is bounded, the integral (19) diverges for $s=s^{*}$ and

$$
\lim _{s \rightarrow s^{*}} \tilde{s}\left(s, x^{0}\right)=+\infty
$$

otherwise there exists a $\tilde{s}^{*}$ such that

$$
\lim _{s \rightarrow s^{*}} \tilde{s}\left(s, x^{0}\right)=\tilde{s}^{*}
$$

In either cases the solutions of the new system satisfies

$$
\lim _{\tilde{s} \rightarrow+\infty} \tilde{U}\left(\tilde{s}, x^{0}\right)=U\left(s^{*}, x^{0}\right)
$$

Moreover $\tilde{s}\left(s, x^{0}\right)$ is invertible with respect to $s$ and we note $S\left(\tilde{s}, x^{0}\right)$ its inverse which satisfies the additional ODE

$$
\dot{S}\left(\tilde{s}, x^{0}\right)=\tilde{\alpha}\left(\tilde{s}, x^{0}\right), S\left(0, x^{0}\right)=0 .
$$

The meaning of (27) is that we have defined modified rays which fill the time-space configuration but correspond to old rays until they reach the caustic. The rays in the old time variable (20) can be recovered using (28) as ((23) again)

$$
U\left(S\left(\tilde{s}, x^{0}\right), x^{0}\right)=\tilde{U}\left(\tilde{s}, x^{0}\right) .
$$

$\mathrm{RR} \mathrm{n}^{\circ} 3786$ 


\subsection{From Lagrangian to Eulerian}

The pathologies of section 3 being fixed (at least in the Lagrangian framework) we derive a Eulerian method from the modified Lagrangian system (24-28).

The new-time Eulerian vector-valued function, denoted $V(\tilde{t}, x)$ is defined by

$$
V\left(\tilde{s}, \tilde{y}\left(\tilde{s}, x^{0}\right)\right)=\tilde{U}\left(\tilde{s}, x^{0}\right) .
$$

The first step is to differentiate (30) with respect to $\tilde{s}$. The chain rule gives for all $j\left(V_{j}, F_{j}\right.$ and $U_{j}^{0}$ are the $j^{\text {th }}$ component of $V, F$ and $U_{j}^{0}$ )

$$
\left\{\begin{array}{c}
\frac{\partial V_{j}}{\partial \tilde{s}}\left(\tilde{s}, \tilde{y}\left(\tilde{s}, x^{0}\right)\right)+\dot{\tilde{y}}\left(\tilde{s}, x^{0}\right) \cdot V_{j, x}\left(\tilde{s}, \tilde{y}\left(\tilde{s}, x^{0}\right)\right)=\ldots \\
\tilde{\alpha}\left(\tilde{s}, x^{0}\right) F_{j}\left(\tilde{s}, V\left(\tilde{s}, \tilde{y}\left(\tilde{s}, x^{0}\right)\right)\right) \\
V_{j}\left(0, x^{0}\right)=U_{j}^{0}\left(x^{0}\right), \forall x^{0}
\end{array}\right.
$$

where we can eliminate $\dot{\tilde{y}}\left(\tilde{s}, x^{0}\right)$ and $\tilde{\alpha}\left(\tilde{s}, x^{0}\right)$ (using (24))

$$
\left\{\begin{array}{c}
\frac{\partial V_{j}}{\partial \tilde{s}}\left(\tilde{s}, \tilde{y}\left(\tilde{s}, x^{0}\right)\right)+G\left(V\left(\tilde{s}, \tilde{y}\left(\tilde{s}, x^{0}\right)\right)\right) H_{p}\left(\tilde{s}, \tilde{y}\left(\tilde{s}, x^{0}\right), \tilde{p}\left(\tilde{s}, x^{0}\right)\right) \cdot V_{j, x}\left(\tilde{s}, \tilde{y}\left(\tilde{s}, x^{0}\right)\right)=\ldots \\
G\left(V\left(\tilde{s}, \tilde{y}\left(\tilde{s}, x^{0}\right)\right)\right) F_{j}\left(\tilde{s}, V\left(\tilde{s}, \tilde{y}\left(\tilde{s}, x^{0}\right)\right)\right) \\
V_{j}\left(0, x^{0}\right)=U_{j}^{0}\left(x^{0}\right), \forall x^{0}
\end{array}\right.
$$

Under the new time parameterization, the field of rays $\tilde{y}\left(\tilde{s}, x^{0}\right)$ advantageously avoid caustics, remains single valued and defined everywhere. In particular, $\tilde{\alpha}\left(\tilde{s}, x^{0}\right)$ may tend to 0 if the corresponding ray heads towards a caustic but will never actually take a zero value.

The definition (30) is therefore proper, the function $V(\tilde{t}, x)$ is single-valued and correctly defined. We can write the system (32) in Eulerian coordinates $(\tilde{t}, x)$ (instead of the Lagrangian $\left(\tilde{s}, \tilde{y}\left(\tilde{s}, x^{0}\right)\right)$ ); for all $j$

$$
\left\{\begin{array}{c}
\frac{\partial V_{j}}{\partial \tilde{t}}(\tilde{t}, x)+G(V(\tilde{t}, x)) H_{p}\left(\tilde{t}, x, V_{2}(\tilde{t}, x)\right) \cdot V_{j, x}(\tilde{t}, x)=\ldots \\
G(V(\tilde{t}, x)) F_{j}(\tilde{t}, V(\tilde{t}, x)) \\
V_{j}(0, x)=U_{j}^{0}(x), \forall x
\end{array}\right.
$$

where $\tilde{p}\left(\tilde{s}, x^{0}\right)$ was eliminated using the identity $V_{2}\left(\tilde{s}, \tilde{y}\left(s, x^{0}\right)\right)=\tilde{p}\left(\tilde{s}, x^{0}\right)$ (from (30) 2920$)$.

Because of our new non-local time parameterization of the problem (19), (45) (next section) does not simplify and we have to solve the full Eulerian system (33). An Eulerian variable $T(\tilde{t}, x)$ for the old time can be defined likewise

$$
T\left(\tilde{s}, \tilde{y}\left(\tilde{s}, x^{0}\right)\right)=S\left(\tilde{s}, x^{0}\right)
$$

and satisfies the Eulerian transport equation:

$$
\left\{\begin{array}{c}
\frac{\partial T}{\partial \tilde{t}}(\tilde{t}, x)+G(V(\tilde{t}, x)) H_{p}\left(\tilde{t}, x, V_{2}(\tilde{t}, x)\right) \cdot T_{x}(\tilde{t}, x)=G(V(\tilde{t}, x)), \\
T(0, x)=0, \forall x .
\end{array}\right.
$$

It is used to recover the Eulerian solution in the old time setting, called $W(t, x)\left(\right.$ set $\left.W\left(s, y\left(s, x^{0}\right)\right)=U\left(s, x^{0}\right)\right)$ using the identity

$$
W(T(\tilde{t}, x), x)=V(\tilde{t}, x) .
$$

This Eulerian system of coupled non-linear transport equations (33-35) is used for capturing caustics. Before the numerical discussion and the presentation of our simulation results, we explore in the next section the connection between these new-time Eulerian and Lagrangian variables (which simplify in the classical case to give the relations (6)). 


\subsection{More on Eulerian/Lagrangian correspondence}

We focus on the phase $\phi(\tilde{t}, x)$

$$
\phi\left(\tilde{s}, \tilde{y}\left(\tilde{s}, x^{0}\right)\right)=V_{3}\left(\tilde{s}, \tilde{y}\left(s, x^{0}\right)\right)=\tilde{\varphi}\left(\tilde{s}, x^{0}\right)
$$

The third equation of $(32)$ is

$$
\left\{\begin{array}{c}
\frac{\partial \phi}{\partial \tilde{s}}\left(\tilde{s}, \tilde{y}\left(\tilde{s}, x^{0}\right)\right)+\tilde{\alpha}\left(\tilde{s}, x^{0}\right) H_{p}\left(\tilde{s}, \tilde{y}\left(\tilde{s}, x^{0}\right), \tilde{p}\left(\tilde{s}, x^{0}\right)\right) \cdot \phi_{x}\left(\tilde{s}, \tilde{y}\left(\tilde{s}, x^{0}\right)\right)=\ldots \\
\tilde{\alpha}\left(\tilde{s}, x^{0}\right)\left(\tilde{p}\left(\tilde{s}, x^{0}\right) \cdot H_{p}\left(\tilde{s}, \tilde{y}\left(\tilde{s}, x^{0}\right), \tilde{p}\left(\tilde{s}, x^{0}\right)\right)-H\left(\tilde{s}, \tilde{y}\left(\tilde{s}, x^{0}\right), \tilde{p}\left(\tilde{s}, x^{0}\right)\right)\right) \\
\phi\left(0, x^{0}\right)=\phi^{0}\left(x^{0}\right), \forall x^{0}
\end{array}\right.
$$

The next "classical" step is usually to identify the $p$ component of the bicharacteristics with $\phi_{x}$. It is done by differentiating (38) with respect to $x_{i}^{0}$ (now the $i$ th component of $x^{0}$ ). Without expanding the time derivation on the left hand side, we get :

$$
\left\{\begin{array}{c}
\frac{\partial}{\partial \tilde{s}}\left\{\frac{\partial \tilde{y}}{\partial x_{i}^{0}}\left(\tilde{s}, x^{0}\right) \cdot \phi_{x}\left(\tilde{s}, \tilde{y}\left(\tilde{s}, x^{0}\right)\right)\right\}=\ldots \\
\frac{\partial \tilde{\alpha}}{\partial x_{i}^{0}}\left(\tilde{p}\left(\tilde{s}, x^{0}\right) \cdot H_{p}\left(\tilde{s}, \tilde{y}\left(\tilde{s}, x^{0}\right), \tilde{p}\left(\tilde{s}, x^{0}\right)\right)-H\left(\tilde{s}, \tilde{y}\left(\tilde{s}, x^{0}\right), \tilde{p}\left(\tilde{s}, x^{0}\right)\right)\right)+ \\
\tilde{\alpha}\left(\tilde{s}, x^{0}\right) \frac{\partial}{\partial x_{i}^{0}}\left\{\tilde{p}\left(\tilde{s}, x^{0}\right) \cdot H_{p}\left(\tilde{s}, \tilde{y}\left(\tilde{s}, x^{0}\right), \tilde{p}\left(\tilde{s}, x^{0}\right)\right)-H\left(\tilde{s}, \tilde{y}\left(\tilde{s}, x^{0}\right), \tilde{p}\left(\tilde{s}, x^{0}\right)\right)\right\}
\end{array}\right.
$$

It is possible to simplify the right hand side (the calculations are easier to perform in terms of $(\dot{\tilde{y}}, \dot{\tilde{p}})$ ) and we obtain

$$
\left\{\begin{array}{c}
\frac{\partial}{\partial \tilde{s}}\left\{\frac{\partial \tilde{y}}{\partial x_{i}^{0}}\left(\tilde{s}, x^{0}\right) \cdot \phi_{x}\left(\tilde{s}, \tilde{y}\left(\tilde{s}, x^{0}\right)\right)\right\}=\ldots \\
\left.\frac{\partial}{\partial \tilde{s}}\left\{\frac{\partial \tilde{y}}{\partial x_{i}^{0}}\left(\tilde{s}, x^{0}\right) \cdot \tilde{p}\left(\tilde{s}, x^{0}\right)\right\}-\frac{\partial \tilde{\alpha}}{\partial x_{i}^{0}}\left(\tilde{s}, x^{0}\right) H\left(\tilde{s}, \tilde{y}\left(\tilde{s}, x^{0}\right), \tilde{p}\left(\tilde{s}, x^{0}\right)\right)\right)
\end{array}\right.
$$

It can be checked that

$$
\frac{\partial}{\partial \tilde{s}}\left\{H\left(\tilde{s}, \tilde{y}\left(\tilde{s}, x^{0}\right), \tilde{p}\left(\tilde{s}, x^{0}\right)\right)\right\}=0
$$

and after subtracting

$$
\int_{0}^{\tilde{s}} \frac{\partial \tilde{\alpha}}{\partial x_{i}^{0}}\left(\tau, x^{0}\right) d \tau \frac{\partial}{\partial \tilde{s}}\left\{H\left(\tilde{s}, \tilde{y}\left(\tilde{s}, x^{0}\right), \tilde{p}\left(\tilde{s}, x^{0}\right)\right)\right\}
$$

to $(40)$ we obtain

$$
\left\{\begin{array}{c}
\frac{\partial}{\partial \tilde{s}}\left\{\frac{\partial \tilde{y}}{\partial x_{i}^{0}}\left(\tilde{s}, x^{0}\right) \cdot \phi_{x}\left(\tilde{s}, \tilde{y}\left(\tilde{s}, x^{0}\right)\right)\right\}=\ldots \\
\frac{\partial}{\partial \tilde{s}}\left\{\frac{\partial \tilde{y}}{\partial x_{i}^{0}}\left(\tilde{s}, x^{0}\right) \cdot \tilde{p}\left(\tilde{s}, x^{0}\right)-\int_{0}^{\tilde{s}} \frac{\partial \tilde{\alpha}}{\partial x_{i}^{0}}\left(\tau, x^{0}\right) d \tau H\left(\tilde{s}, \tilde{y}\left(\tilde{s}, x^{0}\right), \tilde{p}\left(\tilde{s}, x^{0}\right)\right)\right\} .
\end{array}\right.
$$

We verify that the initial values $(\tilde{s}=0)$ for the quantities inside braces match (the initialization of $\varphi$ and $p$ is precisely designed for) and then, along a ray and for all $i$, the following equation

$$
\left\{\begin{array}{c}
\frac{\partial \tilde{y}}{\partial x_{i}^{0}}\left(\tilde{s}, x^{0}\right) \cdot \phi_{x}\left(\tilde{s}, \tilde{y}\left(\tilde{s}, x^{0}\right)\right)=\ldots \\
\frac{\partial \tilde{y}}{\partial x_{i}^{0}}\left(\tilde{s}, x^{0}\right) \cdot \tilde{p}\left(\tilde{s}, x^{0}\right)-\int_{0}^{\tilde{s}} \frac{\partial \tilde{\alpha}}{\partial x_{i}^{0}}\left(\tau, x^{0}\right) d \tau H\left(\tilde{s}, \tilde{y}\left(\tilde{s}, x^{0}\right), \tilde{p}\left(\tilde{s}, x^{0}\right)\right)
\end{array}\right.
$$

is satisfied. The no caustic condition $\tilde{\alpha} \neq 0$ ensures that the family of vectors $\left(\frac{\partial \tilde{y}}{\partial x_{i}^{0}}\right)_{i=1 . . d}$ is a basis of $\mathbb{R}^{d}$. Therefore the identification formula

$$
\tilde{p}\left(\tilde{s}, x^{0}\right)=\phi_{x}\left(\tilde{s}, \tilde{y}\left(\tilde{s}, x^{0}\right)\right)+H\left(\tilde{s}, \tilde{y}\left(\tilde{s}, x^{0}\right), \tilde{p}\left(\tilde{s}, x^{0}\right)\right) \sum_{i}\left\{\int_{0}^{\tilde{s}} \frac{\partial \tilde{\alpha}}{\partial x_{i}^{0}}\left(\tau, x^{0}\right) d \tau \eta_{i}\right\}
$$

holds, with $\eta_{i}$ a vector depending on the coordinates of the usual orthonormal basis vectors in $\left(\frac{\partial \tilde{y}}{\partial x_{i}^{0}}\right)_{i=1 . . d}$.

$\mathrm{RR} \mathrm{n}^{\circ} 3786$ 
The classical results of section 2.2 and the relations (6) are now easily established. We simply by notice that the initial Lagrangian method (21) is recovered by setting $\tilde{\alpha} \equiv 1$ in (24) (and then $\frac{\partial \tilde{\alpha}}{\partial x_{i}^{0}} \equiv 0$ ). We also find back the no caustic condition $\alpha \neq 0$ when passing from (44) to (45). In this case the phase equation (38) decouples from the rest of system (32) and we recover the system of Hamilton-Jacobi/transport equation (5-10) in $\phi$ and $\delta$.

\subsection{1-D Numerical results}

We perform our 1-D test on the same example as in section 3.3. We first solve the ODE system (24). The new-time rays $\tilde{y}\left(\tilde{s}, x^{0}\right)$ are displayed in figure 9 . They rapidly reach the caustic and are time asymptotically stationaries.

On the same figure are drawn the color level curves of the new-time phase $\phi(\tilde{t}, x)$. As in section 3 , we solved the system (33-35) on a Cartesian grid using a second order ENO scheme in space and a adaptive third order Runge-Kutta in time.

We now deal with a set of transport equations with advecting field $G(V(\tilde{t}, x)) H_{p}\left(\tilde{t}, x, V_{2}(\tilde{t}, x)\right)$; so we just test for the sign of this quantity to decide whether to upwind in the positive or negative $x$ direction. The phase behaves as the ray field and becomes constant in time at the caustic. As predicted by (45), when $\tilde{\alpha}$ is constant (for $x \leq 0.5$ ) the fronts and the rays are normal. When $\tilde{\alpha}$ decreases and the second component on the left hand side of $(45)$ is non zero, It is not true anymore.

As explained in sections 4.2 and 4.3 we can recover the old time solution living before the caustic using (29) and (36). The old time is the solution of equation (35) (see figure 10) and also becomes constant in time (stationary) at the caustic. So, when we map back in the $s$ and $t$ variables the solutions in figure 9 (see figure 11), the time transformation crushes down the solution above the caustic and accurately determines both the caustic location, the dark zone and the phase associated to solid rays. One must also compare $\beta$, the Eulerian marker for the caustic (see figure 12 and compare it to figure 8).

We want to emphasize that this method provides an easy and convenient way of adaptively refine the meshing near caustics. We use a regular Cartesian grid in space and time $(\tilde{t}, x)$. The grid points are denoted $\left(\tilde{t}_{j}, x_{i}\right)$. Figure 13 zooms on these grid points mapped back in the old time configuration. That is we plot a dot at points $\left(T\left(\tilde{t}_{j}, x_{i}\right), x_{i}\right)$. They concentrate near the caustic and it explains in particular why the solution of figure 12 is much smoother and precise than in figure 8 where the time step is fixed. Remark that it is possible to use $\alpha$ for refining locally the space grid in order to maintain a constant CFL with respect to the old time configuration. The space grid mesh would then asymptotically go to 0 as we approach the caustic. Then the spatial resolution of the caustic could be arbitrarily accurate.

\subsection{Remark on the choice of $\tilde{s}(s)$}

As can be observed on the 1-D numerical results and even though the integral (19) diverges in this case, we were able to recover the stationary solution rapidly (in terms of $\tilde{s}$ ). This is because $\alpha$ behaves in this case as a $O\left(s-s^{*}\right)$ (see section 4.1). This means that $\tilde{s}(s)$ is like a $\log$ of $s$ near the caustic or equivalently that $S(\tilde{s})$ will reach $s^{*}$ (the caustic-reaching time) exponentially fast even though it is slowed by our change of variable.

The 2-3 dimensional numerical test we carried exhibit the same behavior. It must be said that this could be checked (after maybe lengthy computations) for the different types of caustics on the generic classification of these objects (fold and cusp in 2-D, more in 3-D).

We finally stress that (19) is the simplest possible definition for $\tilde{s}(s)$ but any power of $\alpha$ or even some non-linear monotone function of it can be used.

\section{2-3 Dimensional results}

Our Hamiltonian function is now defined for $(y, p) \in \mathbb{R}_{y}^{d} \times \mathbb{R}_{p}^{d}(d=2,3)$ by

$$
H(y, p)=\sqrt{\|p\|^{2}+N(y)}-1 .
$$


This Hamiltonian arises from the high frequency asymptotic of Maxwell Equation. It is used to modelize laser beam propagation in a plasma of electronic density $N$ [13] [20]. We have used an Osher/Godunov scheme for the computation of the viscosity solution and a Van-Leer Slope limiter scheme for our new-time system of transport equations (33-35). The time discretization is explicit and first order.

\section{$5.1 \quad 2-\mathrm{D}$}

The spatial domain is limited to $\left.y=\left(x_{1}, x_{2}\right) \in\right] 0,1[\times] 0,1[$. The space discretization is uniform throughout this section and we used a $50 \times 50$ points grid. The density $N(y)$ is given by

$$
\left\{\begin{array}{c}
N\left(x_{1}, x_{2}\right)=0, \text { for } x_{1}+x_{2} \leq 1.1 \\
N\left(x_{1}, x_{2}\right)=2 *\left(x_{1}+x_{2}-1.1\right)^{2}, \text { else. }
\end{array}\right.
$$

We enforce an in-going plane wave boundary condition on the $x_{1}=0$ and $x_{2}=0$ boundaries of our time-space cube

$$
\left\{\begin{array}{l}
\left(\phi_{x_{1}}, \phi_{x_{2}}\right)\left(t, x_{1}=0, x_{2}\right)=(\cos \theta, \sin \theta), \forall t, \forall x_{2}, \\
\left(\phi_{x_{1}}, \phi_{x_{2}}\right)\left(t, x_{1}, x_{2}=0\right)=(\cos \theta, \sin \theta), \forall t, \forall x_{1},
\end{array}\right.
$$

and out-going boundary conditions elsewhere. We also specify a compatible initial condition

$$
\phi^{0}\left(x_{1}, x_{2}\right)=\cos \theta * x_{1}+\sin \theta * x_{2} .
$$

As in the 1-D case, both the initial conditions and the boundary conditions correspond to rays flowing in the domain. As can be observed on the rays (figure 14) the outgoing boundary condition on $x_{2}=0$ is clearly not adapted to our ray solutions as rays first flow out and then flow in through this boundary. This induces a new pollution effect on the caustic which can again be related to "diffracted rays" discussed earlier. To avoid this problem we have used the classical technique of enlarging the domain to confine these errors in an outer layer. This layer has been removed in all the figures we present.

After some time (approximately $1 \mathrm{~s}$.) the only remaining contribution to the Lagrangian solution comes from the boundary. The boundary condition being stationary, we obtain a stationary solution and stationary caustic curve. Its location can be determined analytically and for $\theta=\pi / 24$ (our example) corresponds to the curve $x_{1}+x_{2}=1.66$ (for a density depending only on one coordinate say $X$ along a direction $\vec{V}$ and in any dimension, the caustic hypersurface is analytically given by the equation $N(X)=\cos (\Theta)$ where $\Theta$ is the angle of the incoming plane wave of rays with the vector $\vec{V}$ (see [20]), we also use this formula in the 3-D section).

The projection of the rays, corresponding to the stationary regime, onto space are plotted in figure 14 . In $1-\mathrm{D}$, it trivially corresponds to one time slice and the projection gives a single plain line from $x=0$ to the caustic point and the same reverse dotted line which returns from the caustic. The red crosses again indicate that we have reached a caustic point.

We will focus in this section on the stationary solution and show how our method recovers the caustic curve. The results are in some sense similar to what we obtained in section 4.4. In both cases we recover a caustic curve for a two dimensional Hamiltonian : time plus one space dimension in one case and two space dimension but a stationary solution in the other.

The pollution effect described previously on the Eulerian solution of the Hamilton-Jacobi equation can be observed on figures 15-18. They show to the contour lines of the phase at successive times and the associated geometrical spreading. The kink effect is present and (as in 1-D figure 7) is not stationary. With this technique there is no hope of recovering the stationary solution and its caustic (indicated by a dark line).

We now proceed exactly as in 1-D, solve our time modified system (33-35) over $6 \mathrm{~s}$. and map back the solution in the old time variable using (36). The stationary solution (see figure 9 ) is crushed down in time and for instance at $t=3 s$. (our old-time slice) the dark side is empty as it should be. The phase is displayed in figure 19 and $\beta$ the geometrical spreading in figure 20. The location of the theoretical caustic is indicated by a black or white line. As in 1-D the pollution effect on the dark side of the caustic has be suppressed as the new time solution cannot cross the caustic.

The contour line of the phase does not reach the caustic because of our graphical software (we use a rather coarse space discretization). Figure 21 is a zoom of figure 20 near the caustic. It shows that our method is exact up to the space discretization. At the points nearest to the caustic $\beta$ is of order of $10^{-1}$ because its profile is very stiff.

$\mathrm{RR} \mathrm{n}^{\circ} 3786$ 


\section{$5.2 \quad 3-\mathrm{D}$}

The Hamiltonian is unchanged (given by (46). We are now in a 3 -D domain $\left.y=\left(x_{1}, x_{2}, x_{3}\right) \in\right] 0,1[\times] 0,1[\times] 0,1[$ and the density $N$ is a $3-\mathrm{D}$ generalization of (47)

$$
\left\{\begin{array}{c}
N\left(x_{1}, x_{2}, x_{3}\right)=0, \text { for } a * x_{1}+b * x_{2}+c * x_{3} \leq 2.1 \\
N\left(x_{1}, x_{2}, x_{3}\right)=2 *\left(a * x_{1}+b * x_{2}+c * x_{3}-2.1\right)^{2}, \text { else. }
\end{array}\right.
$$

so that $N$ is really only a function of $a * x_{1}+b * x_{2}+c * x_{3}$ i.e vary in one direction determined by the coefficients $(a, b, c)$. The boundary and initial conditions now depend on two angles $\theta_{1}$ and $\theta_{2}$

$$
\left\{\begin{array}{l}
\left(\phi_{x_{1}}, \phi_{x_{2}}, \phi_{x_{3}}\right)\left(t, x_{1}=0, x_{2}, x_{3}\right)=\left(\cos \theta_{1} * \cos \theta_{2}, \sin \theta_{1} * \cos \theta_{2}, \sin \theta_{2}\right) \forall t \\
\left(\phi_{x_{1}}, \phi_{x_{2}}, \phi_{x_{3}}\right)\left(t, x_{1}, x_{2}=0, x_{3}\right)=\left(\cos \theta_{1} * \cos \theta_{2}, \sin \theta_{1} * \cos \theta_{2}, \sin \theta_{2}\right) \forall t \\
\left(\phi_{x_{1}}, \phi_{x_{2}}, \phi_{x_{3}}\right)\left(t, x_{1}, x_{2}, x_{3}=0\right)=\left(\cos \theta_{1} * \cos \theta_{2}, \sin \theta_{1} * \cos \theta_{2}, \sin \theta_{2}\right) \forall t
\end{array}\right.
$$

and out-going boundary conditions elsewhere. We again specify a compatible initial condition

$$
\phi^{0}\left(x_{1}, x_{2}, x_{3}\right)=\cos \theta_{1} * \cos \theta_{2} * x_{1}+\sin \theta_{1} * \cos \theta_{2} * x_{2}+\sin \theta_{2} * x_{3} .
$$

As in 1-D and 2-D, the rays are "reflected" from a caustic surface. Its equation can be computed analytically and is given by (see $2-\mathrm{D}$ section)

$$
\begin{gathered}
a * x_{1}+b * x_{2}+c * x_{3}=\ldots \\
2.1+\frac{1}{\sqrt{2}} * \frac{a * \cos \theta_{1} * \cos \theta_{2}+b * \sin \theta_{1} * \cos \theta_{2}+c * \sin \theta_{2}}{\sqrt{a^{2}+b^{2}+c^{2}}} .
\end{gathered}
$$

We show the results for three different sets of parameters. Case $1:(a=1, b=1, c=1)$; Case2 : $(a=1.41, b=1.41, c=0.71)$, Case $3:(a=1.6, b=0.1, c=1.6)$. For all tests $\theta_{1}=\theta_{2}=\pi / 48$. The simulation new-time in $\tilde{s}$ is $9 \mathrm{~s}$. and we present the stationary solution at the old-time $s=4 \mathrm{~s}$.. We use a 50 grid in space but only represent one portion of the domain ( $] 0.5,1[\times] 0.5,1[\times] 0.5,1[)$ where the caustic appears.

Figures 222326273031 show different view points of the isosurfaces of the phase function. The colormap has no meaning here and has be chosen for visibility of the surfaces. As in figure 19 the graphic processing has erased part of the data. The representation of the geometrical spreading $\beta$ (figures 2425282932 33) show that we recover the correct location for the caustic. Again, $\beta$ is very stiff near the caustic and of the order of 0.1 in our grid approximation. The effect of the inconsistent outgoing boundary condition can also be observed.

\section{Conclusion}

Let us first say that caustics are the locus where energy concentrate and a reliable numerical method for the determination of such object should be useful.

The present paper is a companion paper to [5] where an algorithm for the automatic splitting of multi-valued solutions partly based on caustic detection was proposed. Our algorithm is able to capture pieces of caustic (curves or surfaces) on a single branch of the multi-valued solution. When several branches contribute to a single caustic different and more complicated patterns occur. The way of splitting the 2-D generic cuspidal caustic (A3 in the [2] terminology) is explained in [5] (the fold (A2) being a simple sub-case of the cusp). The robustness and accuracy of our caustic capturing method makes it possible to work on the implementation of higher dimensional version of the multi-valued splitting algorithm. In 3-D however, one should carefully study the possibility of carrying a similar splitting on the other specifically 3-D generic caustic (A4 and D4, still [2]).

Caustic are also important theoretical objects and for instance our method could be used to study numerically unstable caustics (focal points).

The "dark zone" pathology raises an interesting question about viscosity solutions and high frequency asymptotics of wave propagation equations. The behavior of the viscosity solution in the areas not covered by classical 
rays indeed correspond, at least in simple cases to diffraction phenomena (see [15]).

Further possible improvement of our method include the implementation of adaptative gridding in space as suggested in section 4.4, the use of robust high order scheme such as WENO schemes (see [23]) and the reduction of the number of equations in our system using simpler methods for computing the geometrical spreading [23] [7].

Finally, the general presentation of the method in section 4.1 (equations (21) (24)) suggests that a similar change of variable may be useful in other free boundary problems.

\section{Acknowledgments}

Part of this work was completed at the "Ecole d'été du CEMRACS", Luminy 1999. The authors also want to acknowledge many useful discussions with O. Lafitte, R. Sentis and Ph. Montarnal.

\section{References}

[1] R. Abgrall and J.-D. Benamou. Big ray tracing and eikonal solver on unstructured grids : Application to the computation of a multi-valued travel-time field in the marmousi model. Geophysics, 64:230-239.

[2] V. I. Arnol'd, S.M. Gusein-Zade, and A.N. Varchenko. Singularities of Differential Maps. Birkhauser, 1986.

[3] G. Barles. Solutions de viscosité des équations de Hamilton-Jacobi. Springer-Verlag, 1994.

[4] J.-D. Benamou. Big ray tracing : Multivalued travel time field computation using viscosity solutions of the eikonal equation. J. Comp. Physics, 128:463-474, 1996.

[5] J.-D. Benamou. Direct solution of multi-valued phase-space solutions for hamilton-jacobi equations. Comm. Pure Appl. Math., 52, 1999.

[6] J.-D. Benamou. Finite-difference computation of 2-d travel-time triplication. rapport sigma 1999, 1999.

[7] J.-D. Benamou and Ph. Montarnal. Equations "géométriques" pour les calcul d'amplitudes d'ondes haute fréquence. preprint, 1999.

[8] Y. Brenier and L.Corrias. A kinetic formulation for multi-branch entropy solutions of scalar conservation laws. Ann. IHP Analyse non-linéaire, 1996.

[9] M.G. Crandall and P.L. Lions. Viscosity solutions of hamilton-jacobi equations. Trans. Amer. Math. Soc., $277: 1-42,1983$.

[10] M.G. Crandall and P.L. Lions. Two approximation solutions of hamilton-jacobi equations. Math. Comp., 43:1-19, 1984.

[11] B. Engquist, E. Fatemi, and S. Osher. Numerical resolution of the high frequency asymptotic expansion of the scalar wave equation. J. Comp. Physics, 120:145-155, 1995.

[12] B. Engquist and O. Runborg. Multi-phase computation in geometrical optics. Tech report, Nada KTH, 1995.

[13] Ph. Montarnal F. Golse, O. Lafitte and R. Sentis. Sur la simulation numérique de la propagation laser. Rapport CEA, 1999.

[14] I.M. Gelfand and S.V Fomin. Calculus of Variation. Prentice-Hall, 1963.

[15] O. Lafitte J.-D. Benamou and I. Solliec. in preparation.

[16] G. Lambare, P. Lucio, and A. Hanyga. Two dimensional multivalued traveltime and amplitude maps by uniform sampling of a ray field. Geophys. J. Int, 125:584-598, 1996.

[17] S. J. Osher and C.W. Shu. High-order essentially nonoscillatory schemes for hamilton-jacobi equations. SIAM J. Numer. Anal., 83:32-78, 1989.

$\mathrm{RR} \mathrm{n}^{\circ} 3786$ 
[18] B. Merryman S. Ruuth and S.J. Osher. A fixed grid method for capturing the motion of self-intersecting interfaces and related pdes. preprint, 1999.

[19] I. Solliec. Résolution numérique de l'équation eikonale issue de l'interaction laser-plasma. Rapport CEA, 1999.

[20] I. Solliec. Sur l'approximation optique d'un faisceau laser. Rapport CEA, 1999.

[21] P. E. Souganidis. Approximation schemes for hamilton-jacobi equations. J. Differential Equations, 59:1-43, 1985.

[22] W. Symes. A slowness matching algorithm for multiple traveltimes. TRIP report, 1996.

[23] W. Symes and J.L. Quian. An adaptive finite difference method for traveltime and amplitudes. TRIP Report Rice U., 1999.

[24] W. Symes, R. Versteeg, A. Sei, and Q. H. Tran. Kirchhoff simulation migration and inversion using finite-difference traveltimes and amplitudes. TRIP tech. Report, Rice U., 1994.

[25] J. Van Trier and W. W. Symes. Upwind finite-difference calculation of traveltimes. Geophysics, 56:812-821, 1991.

[26] V. Vinje, E. Iversen, and H. Gjoystdal. Traveltime and amplitude estimation using wavefront construction. Geophysics, 58:1157-1166, 1993.

[27] L.C. Young. Lecture on the Calculus of Variation and Optimal Control Theory. 1969. 


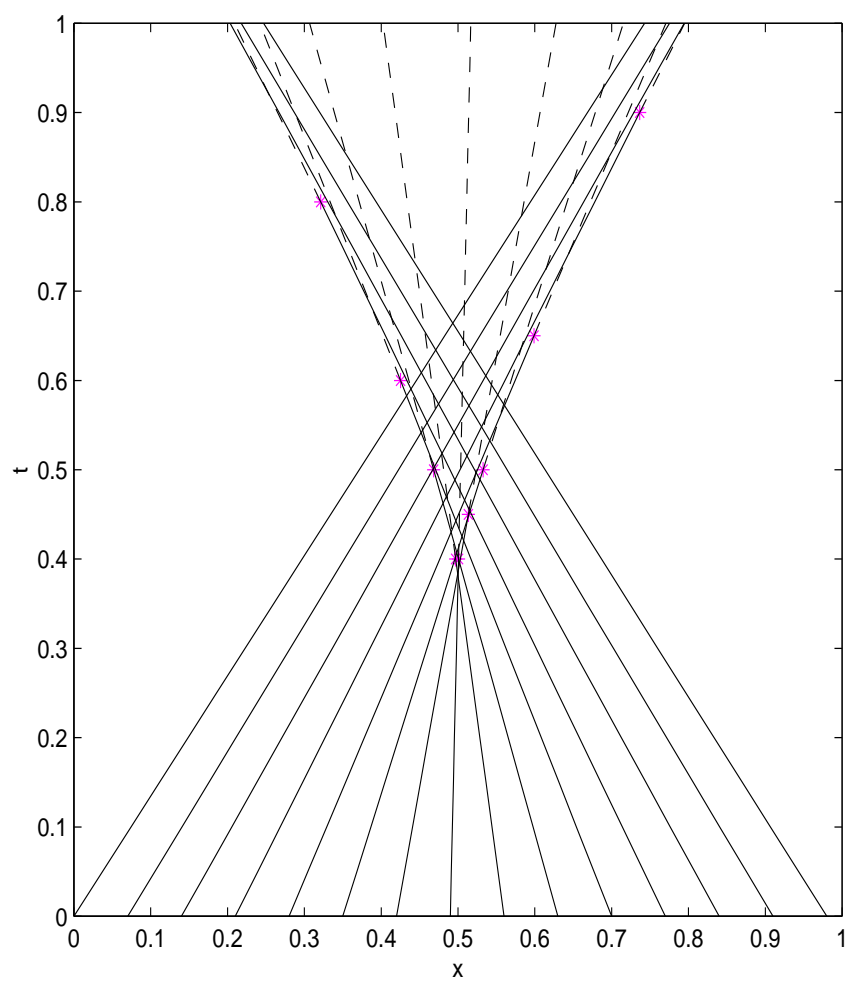

Figure 1: Rays for $n \equiv 1.5, \phi^{0}(x)=-\frac{\sqrt{1+16 *(x-0.5)^{2}}}{2}$. Precaustic part : plain line, Postcaustic part: dashed line. Caustic points : red stars. 


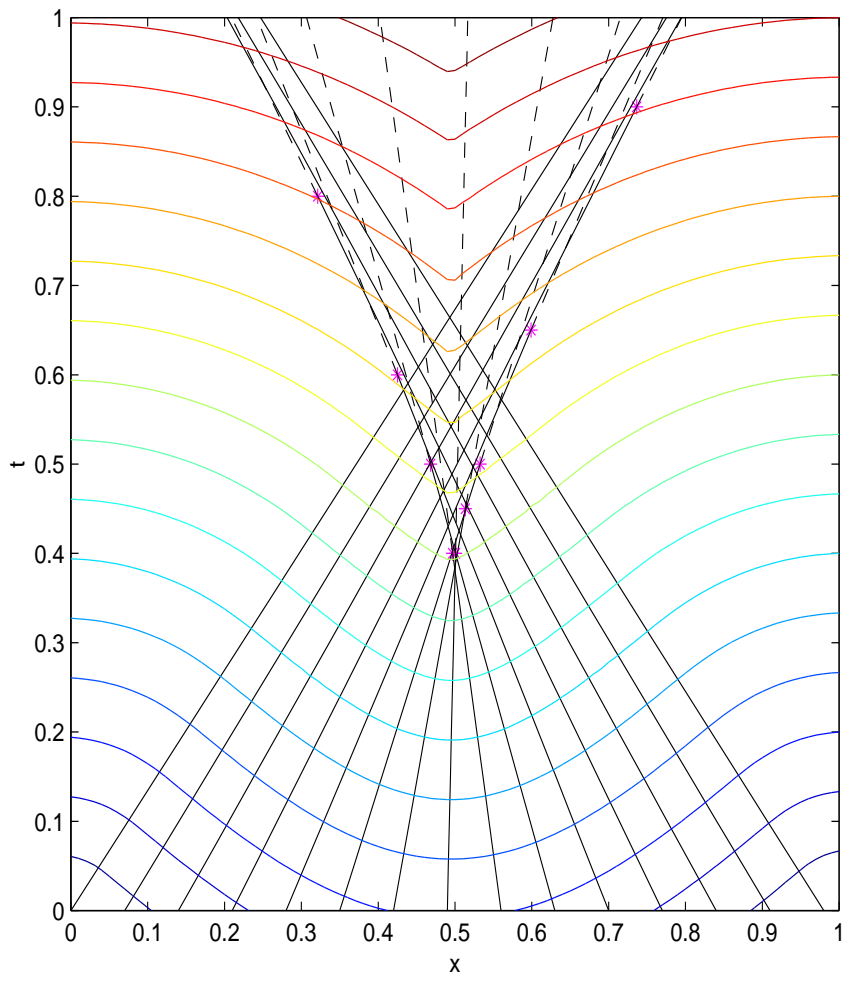

Figure 2: As fig 2 with level curves of the phase added. Color map indicates the value of the phase. 


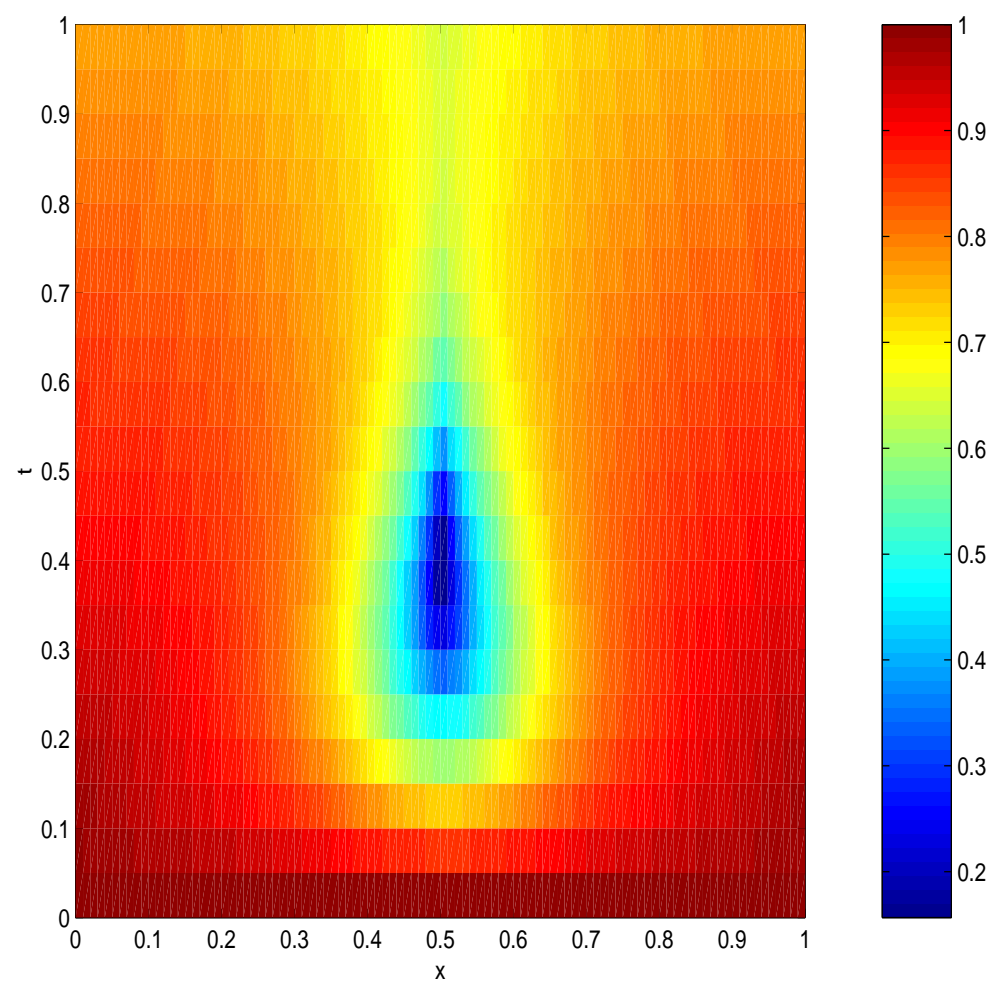

Figure 3: Eulerian geometrical spreading $\beta$ associated to the phase in figure 2. 


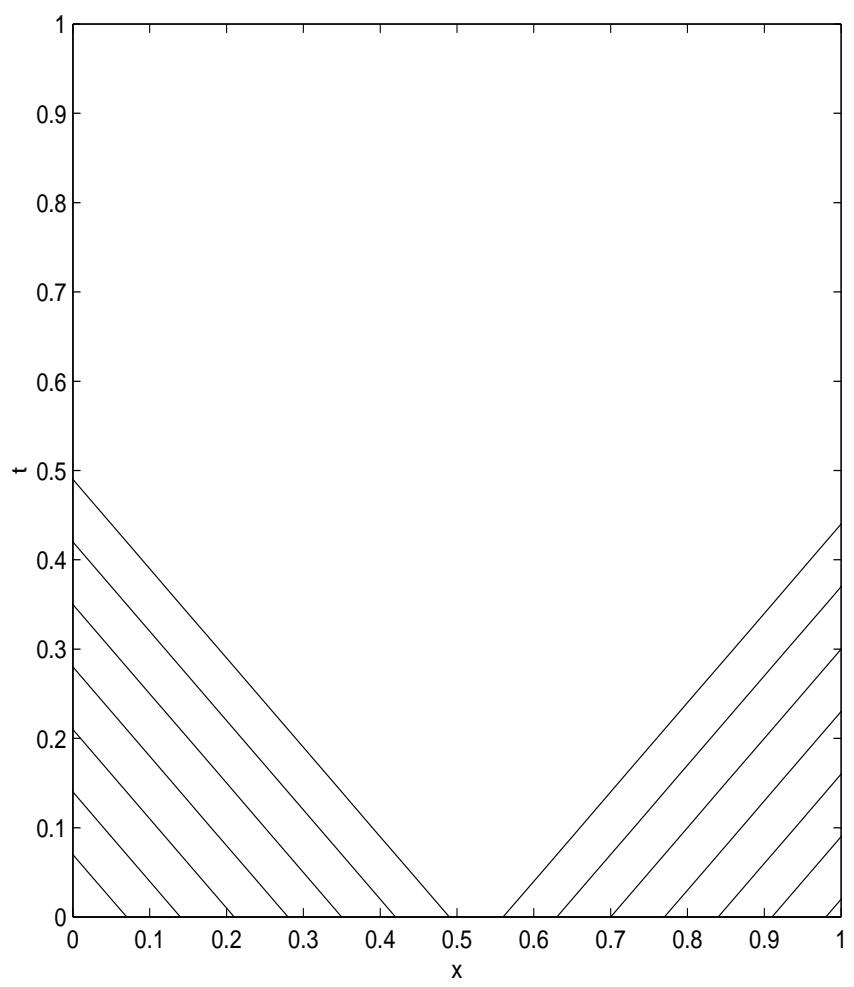

Figure 4: Rays for $n \equiv 3, \phi^{0}(x)=\frac{3 *|x-0.5|}{\sqrt{2}}$. 


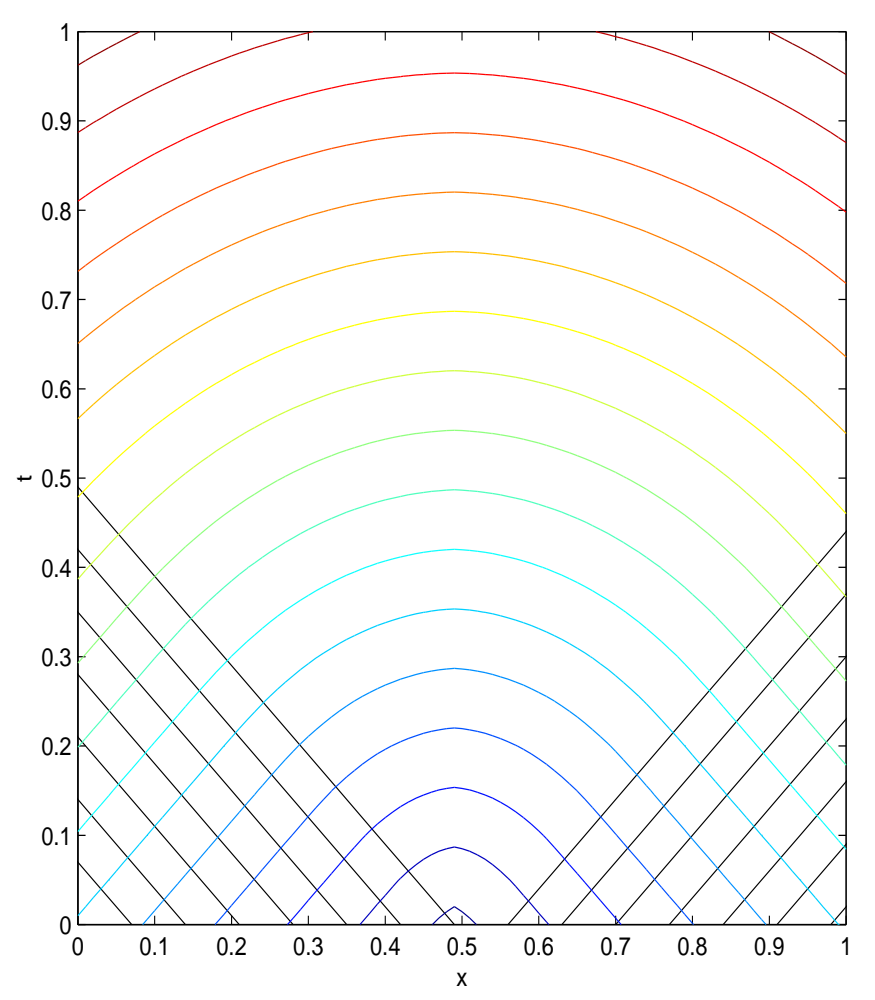

Figure 5: As fig 4 with level curves of the phase added. Color map indicates the value of the phase. 


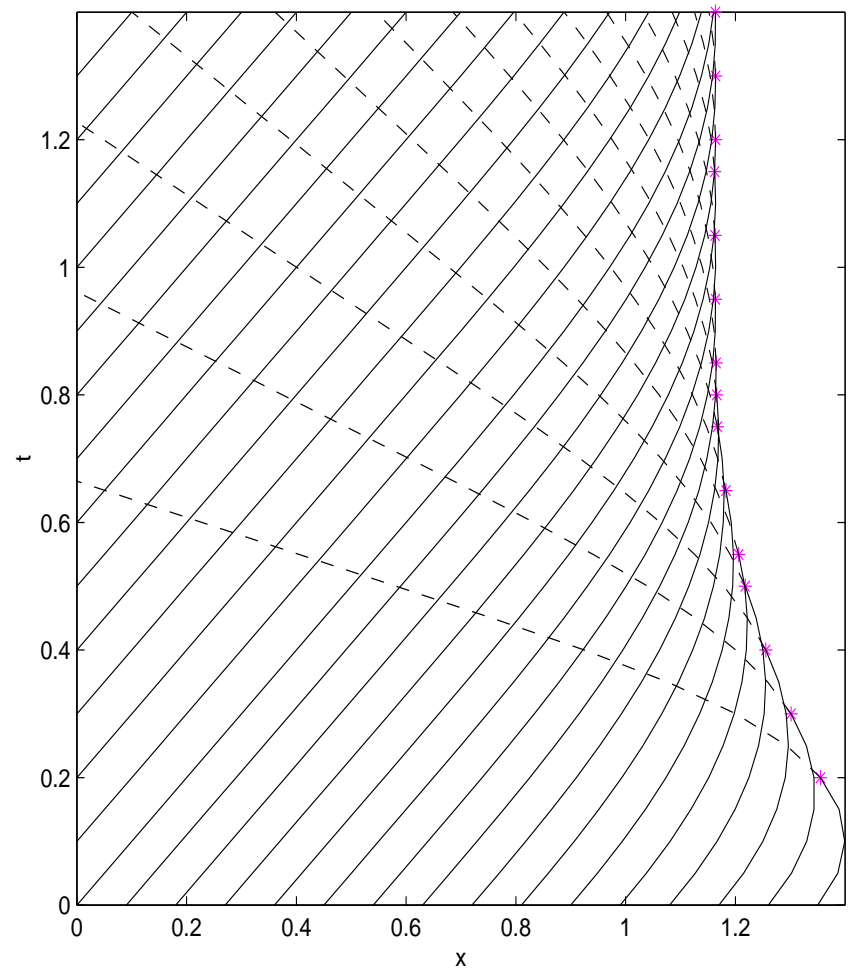

Figure 6: Rays for $n$ as in (16). Initial and boundary conditions as in (17-18). Precaustic part : plain line, Postcaustic part : dashed line. Caustic points : red stars. 


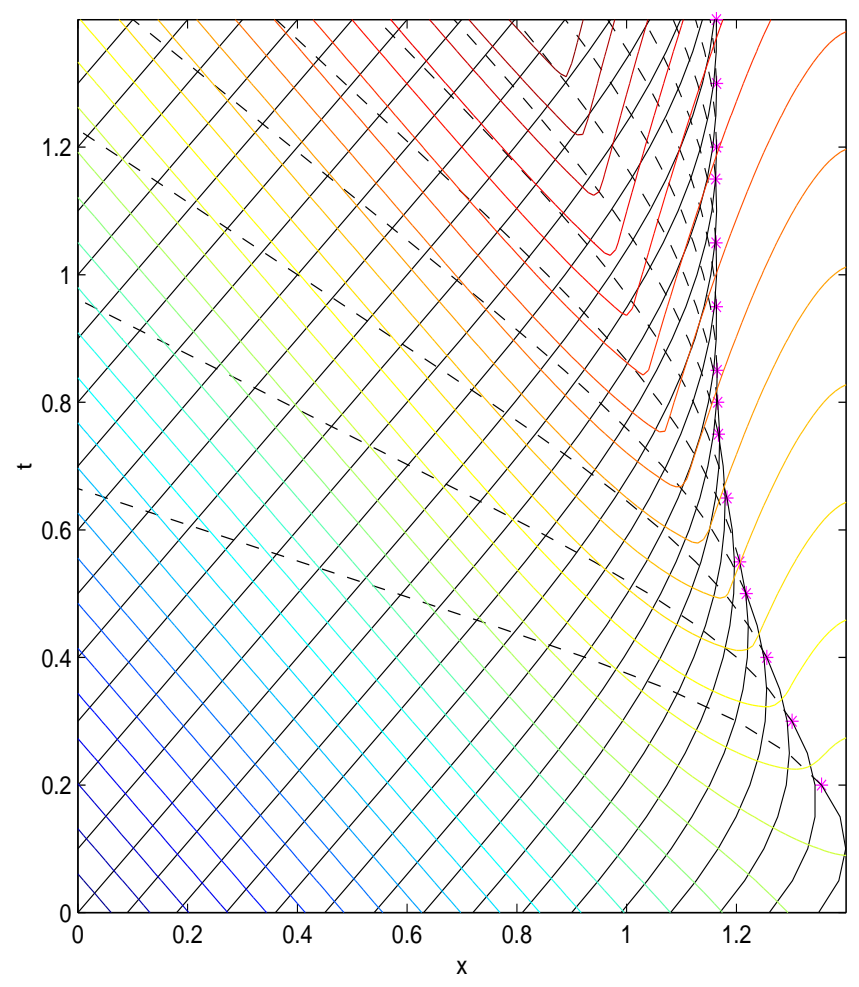

Figure 7: As fig 6 with level curves of the phase added. Color map indicates the value of the phase. 


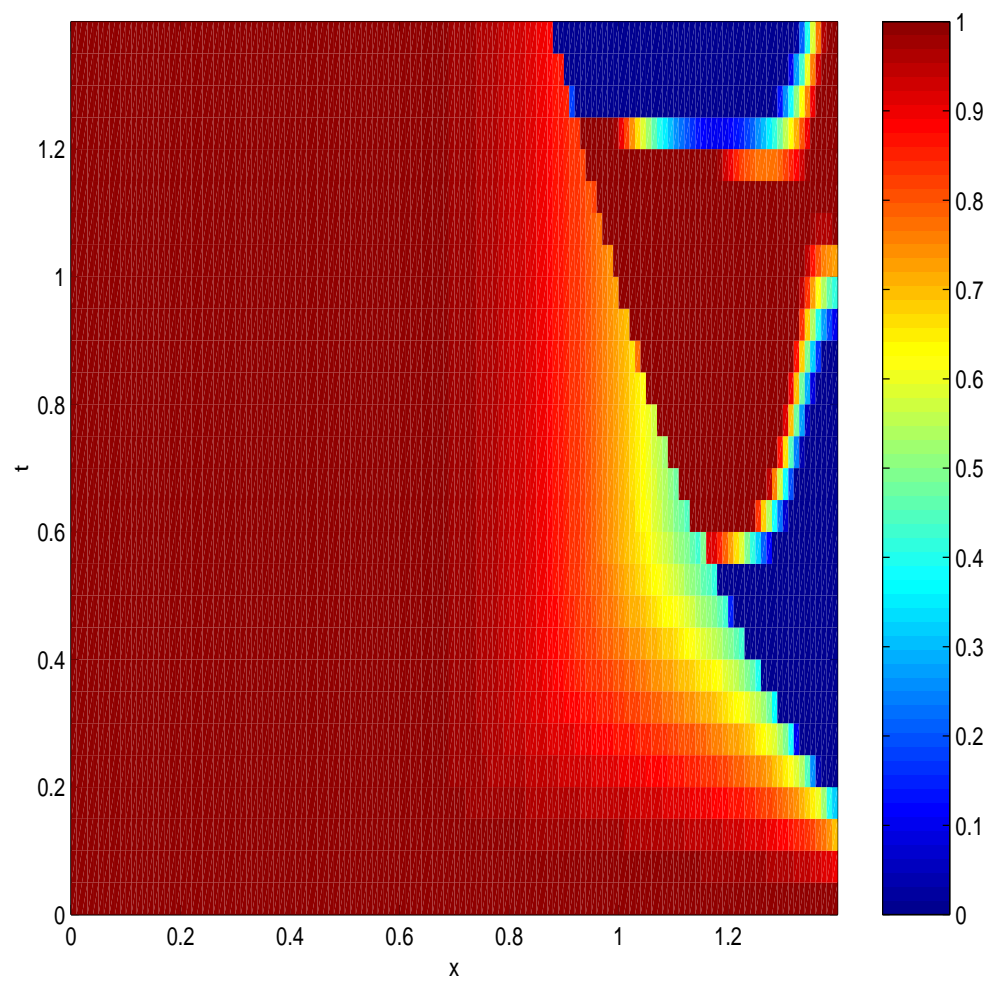

Figure 8: Eulerian geometrical spreading $\beta$ associated to the phase in figure 7 . 


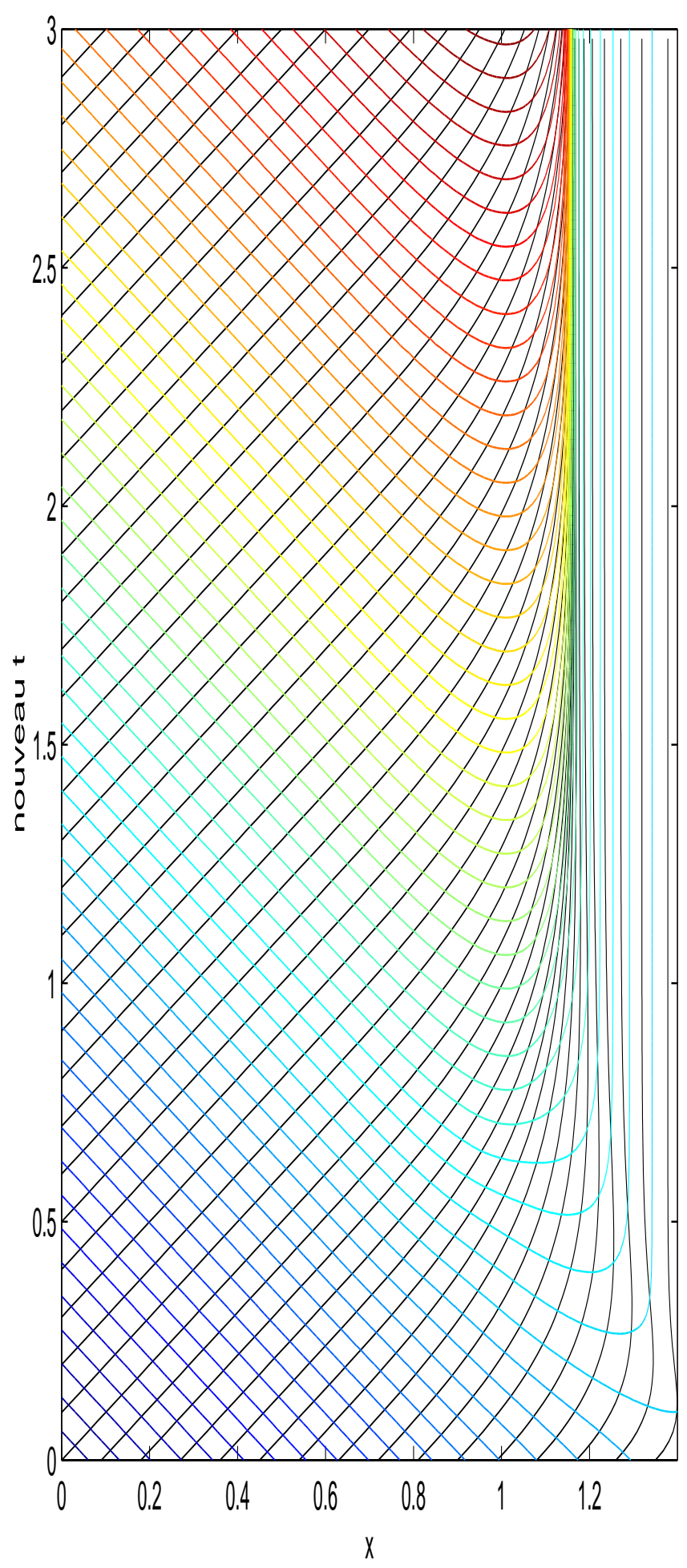

Figure 9: The new time parameterized solution. Rays and level curves of the phase.

$\mathrm{RR} \mathrm{n}^{\circ} 3786$ 


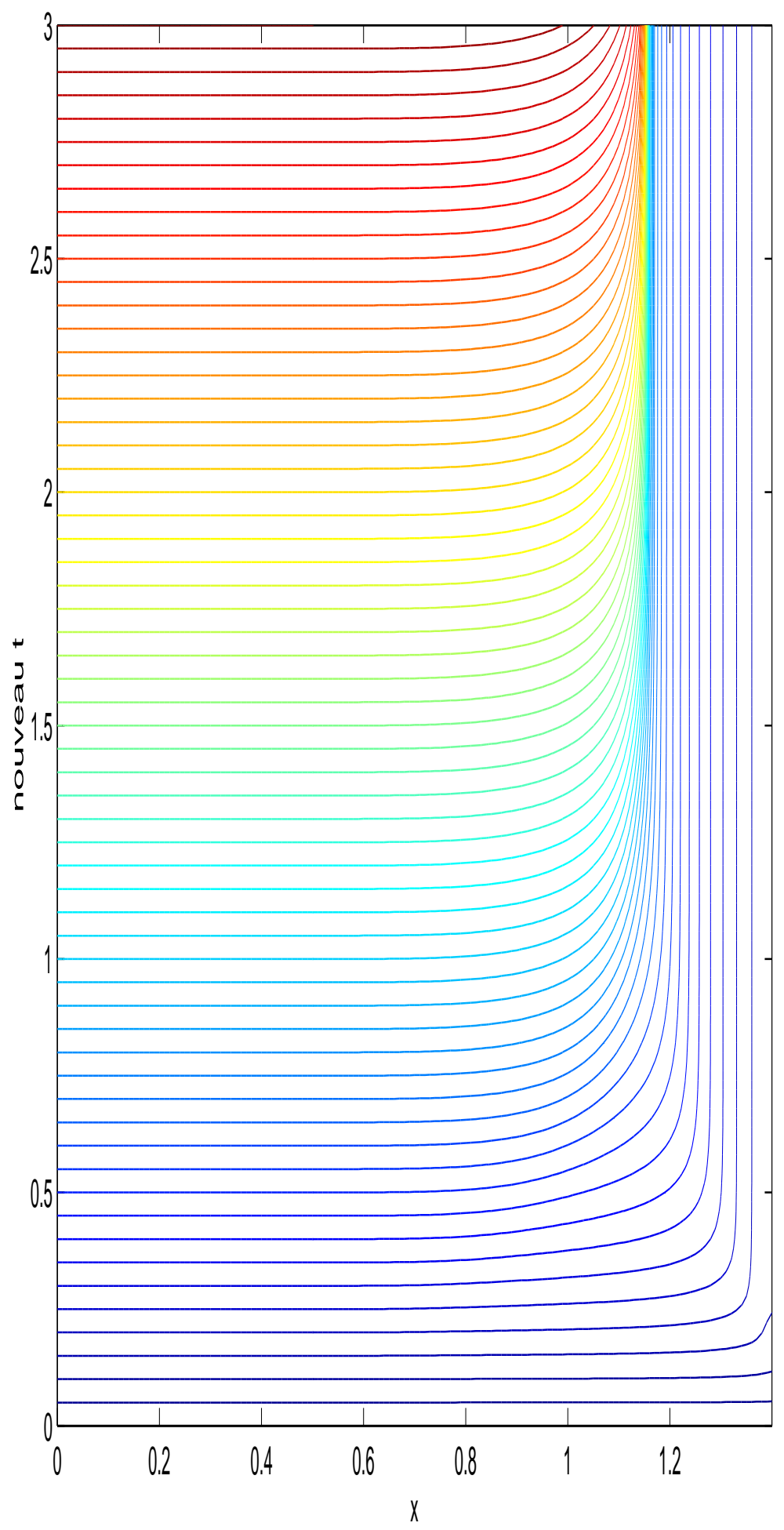

Figure 10: Level curves of the old time variable (solution of (35)). 


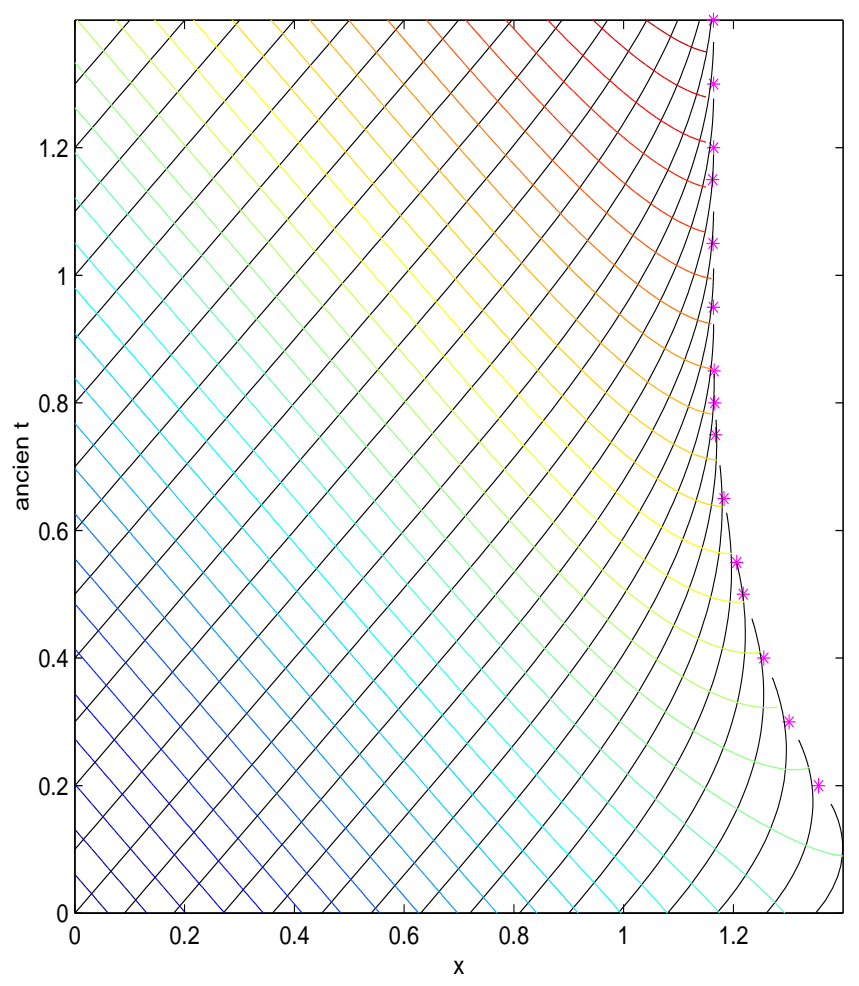

Figure 11: The solutions of figure 9 in the old time variable computed using (35). The red stars indicate the caustic points of figure 6 . 


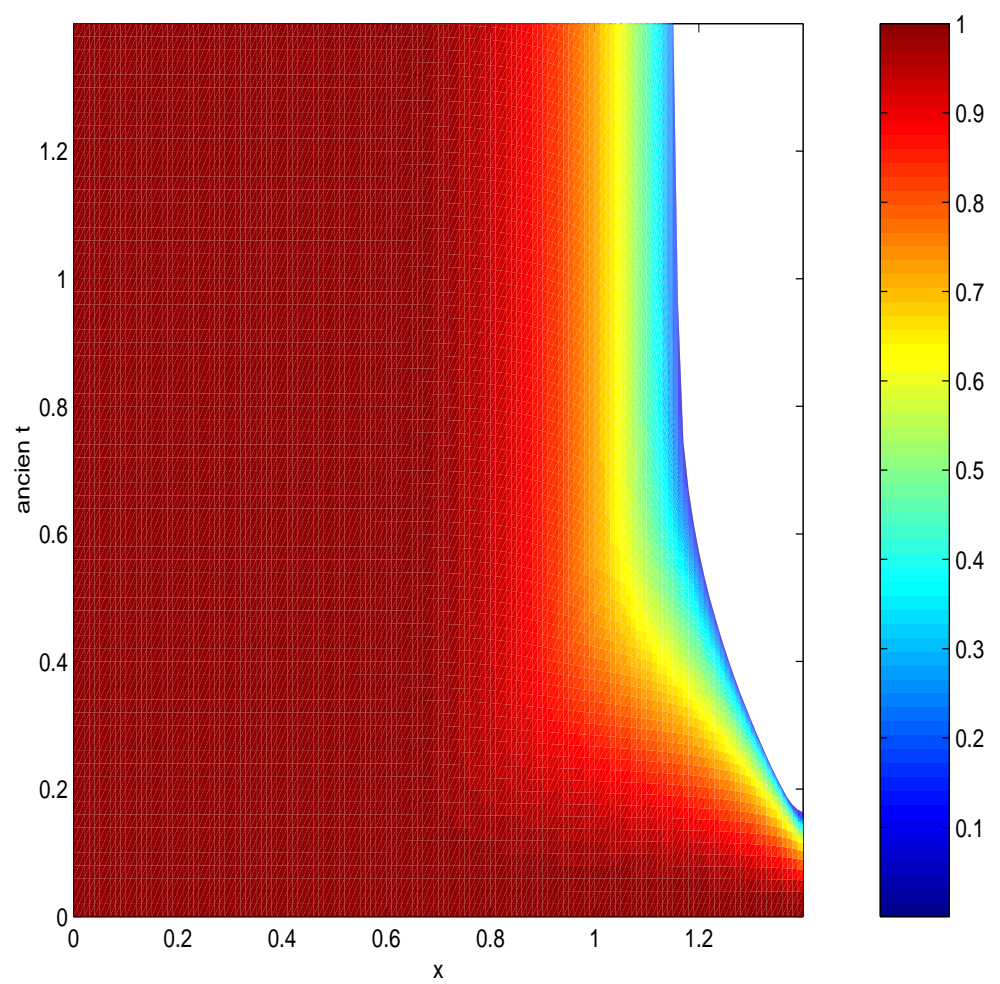

Figure 12: Eulerian geometrical spreading $\beta$ associated to the phase in figure 9 . 


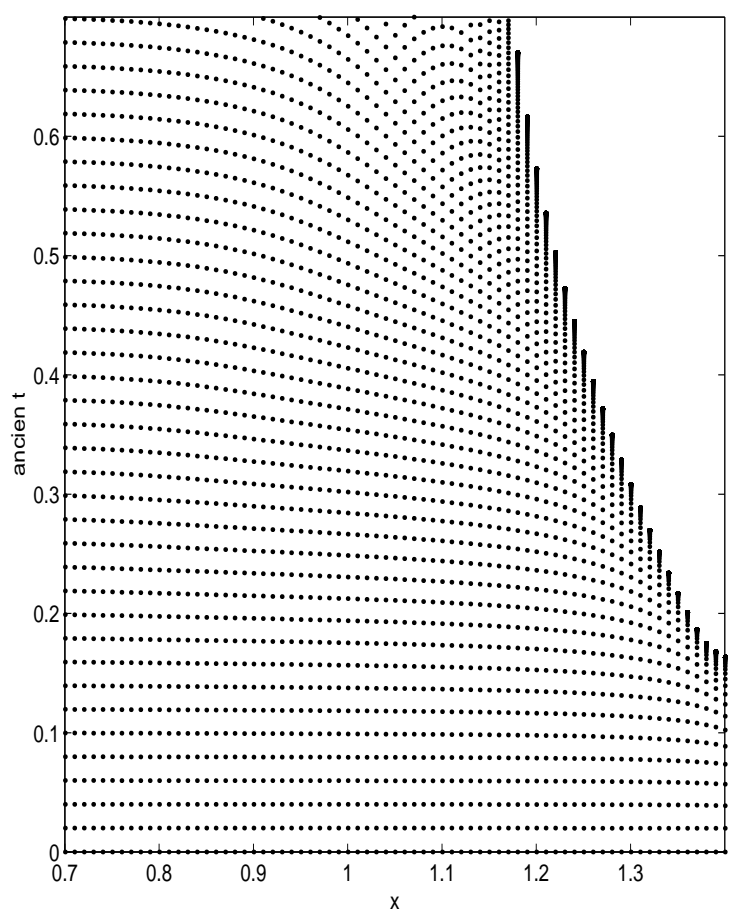

Figure 13: Grid points in the old time configuration. 


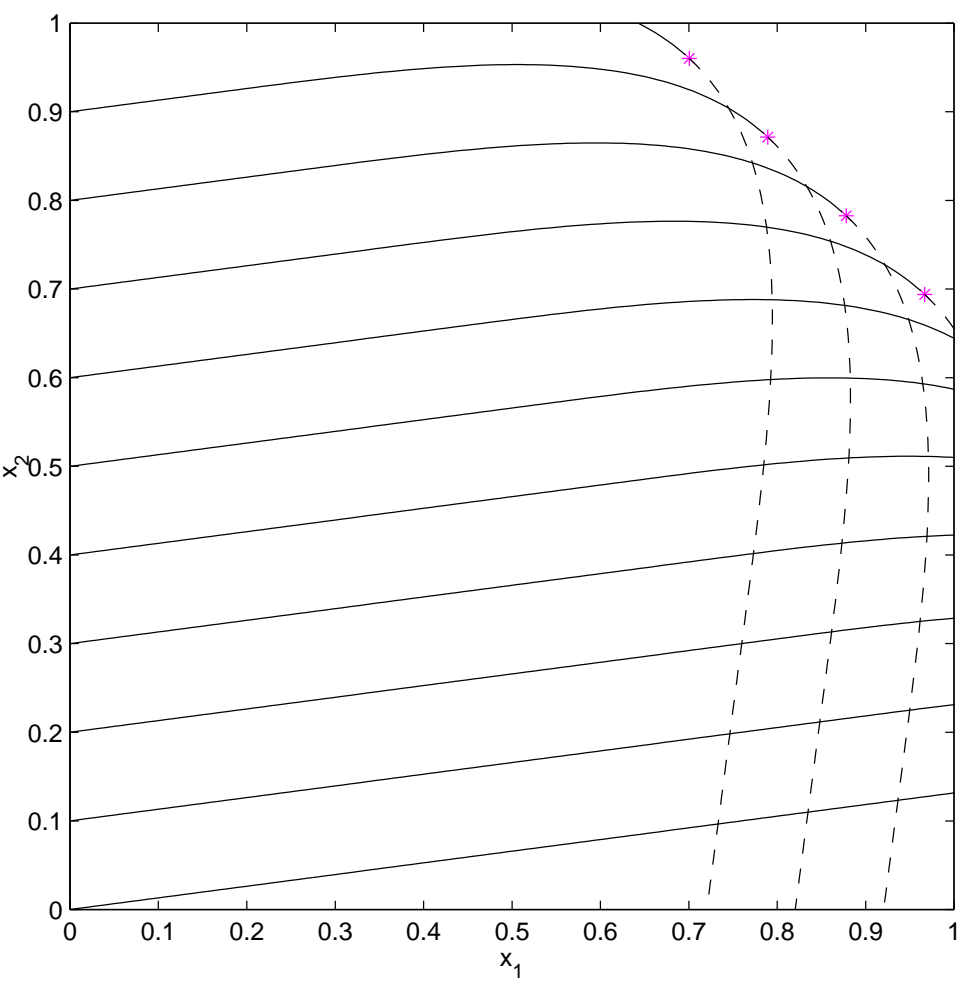

Figure 14: Projection of the rays on the $(x, y)$ plane at the stationary regime in $2-\mathrm{D}$, The red stars indicate the caustic points. 

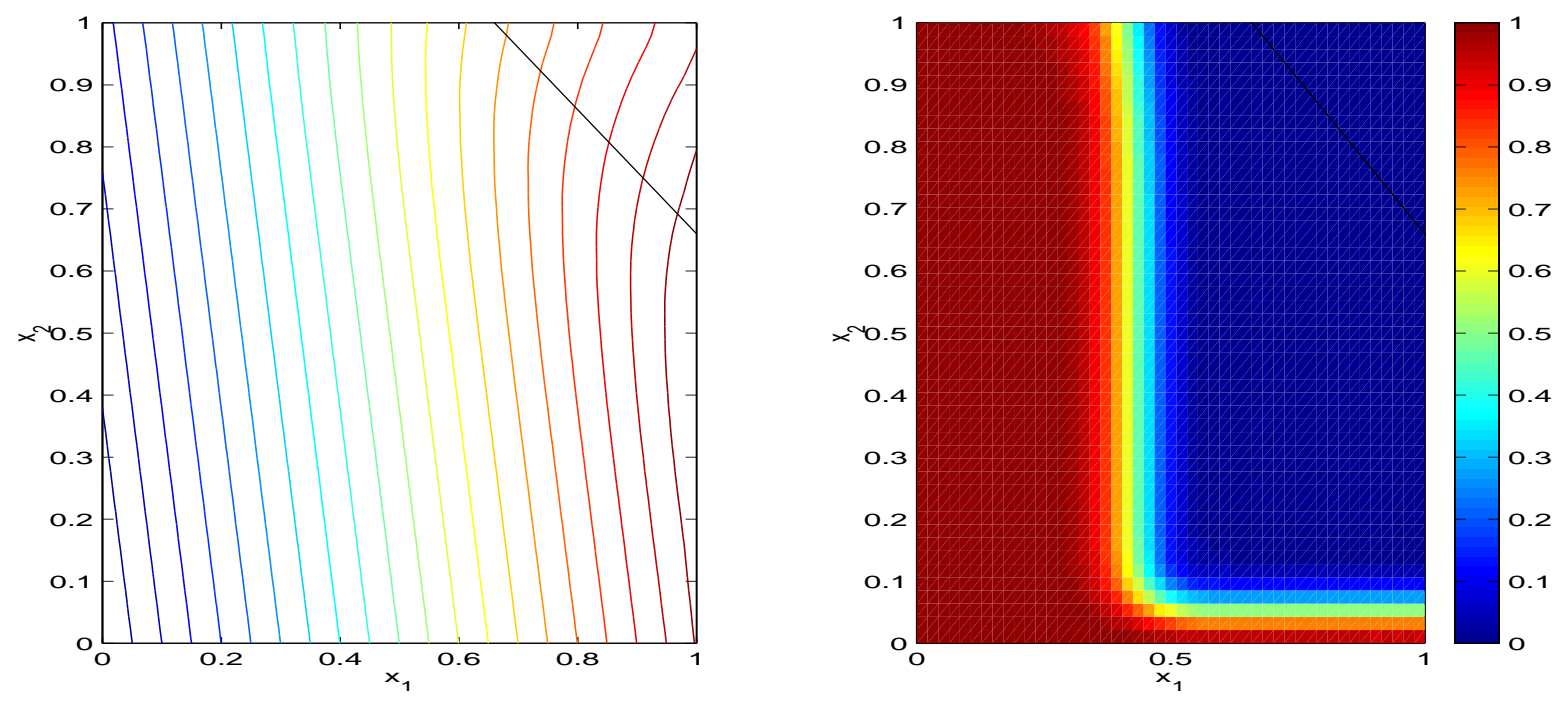

Figure 15: Level lines of the phase (left) and associated geometrical spreading $\beta$ (right) at time $.4 \mathrm{~s}$. The dark line is the theoretical caustic. 

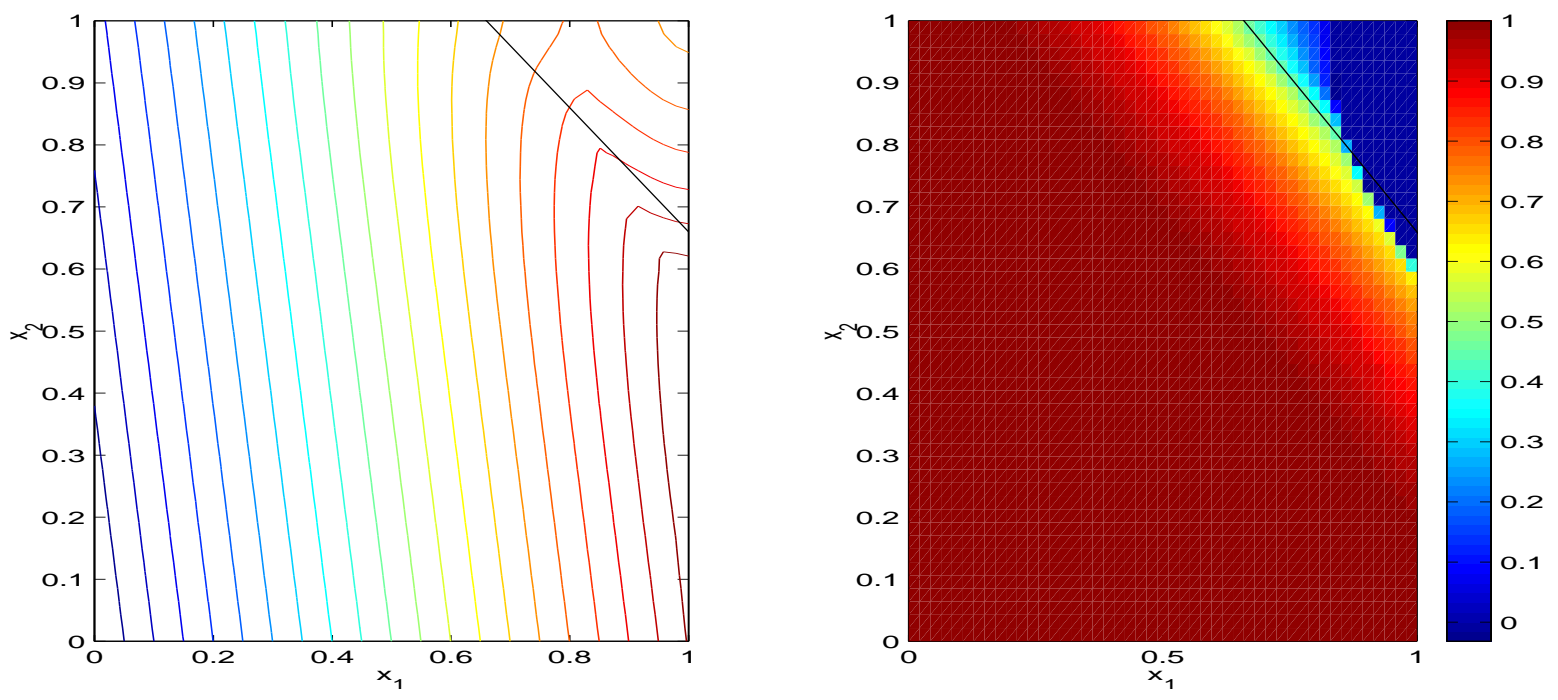

Figure 16: Level lines of the phase (left) and associated geometrical spreading $\beta$ (right) at time $1.2 \mathrm{~s}$. The dark line is the theoretical caustic. 

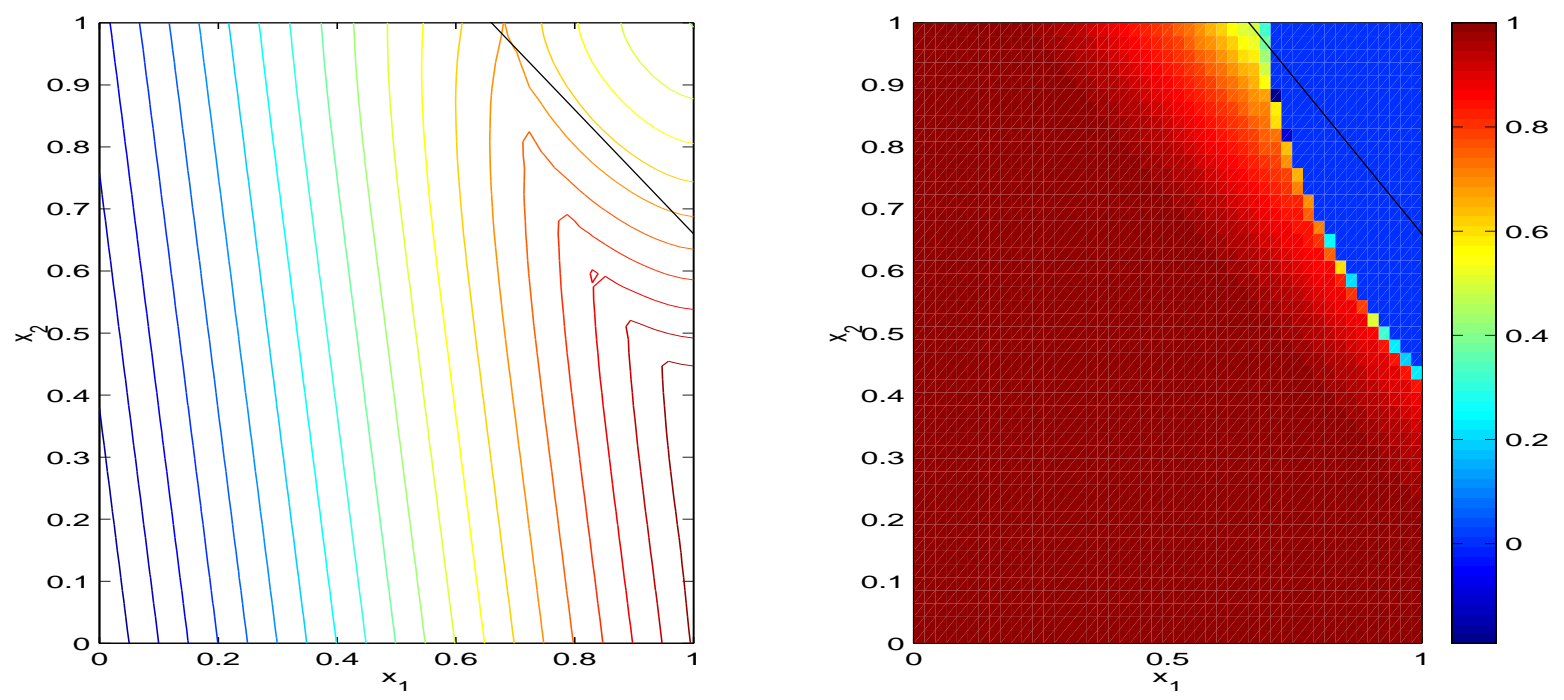

Figure 17: Level lines of the phase (left) and associated geometrical spreading $\beta$ (right) at time $2 \mathrm{~s}$ The dark line is the theoretical caustic. 

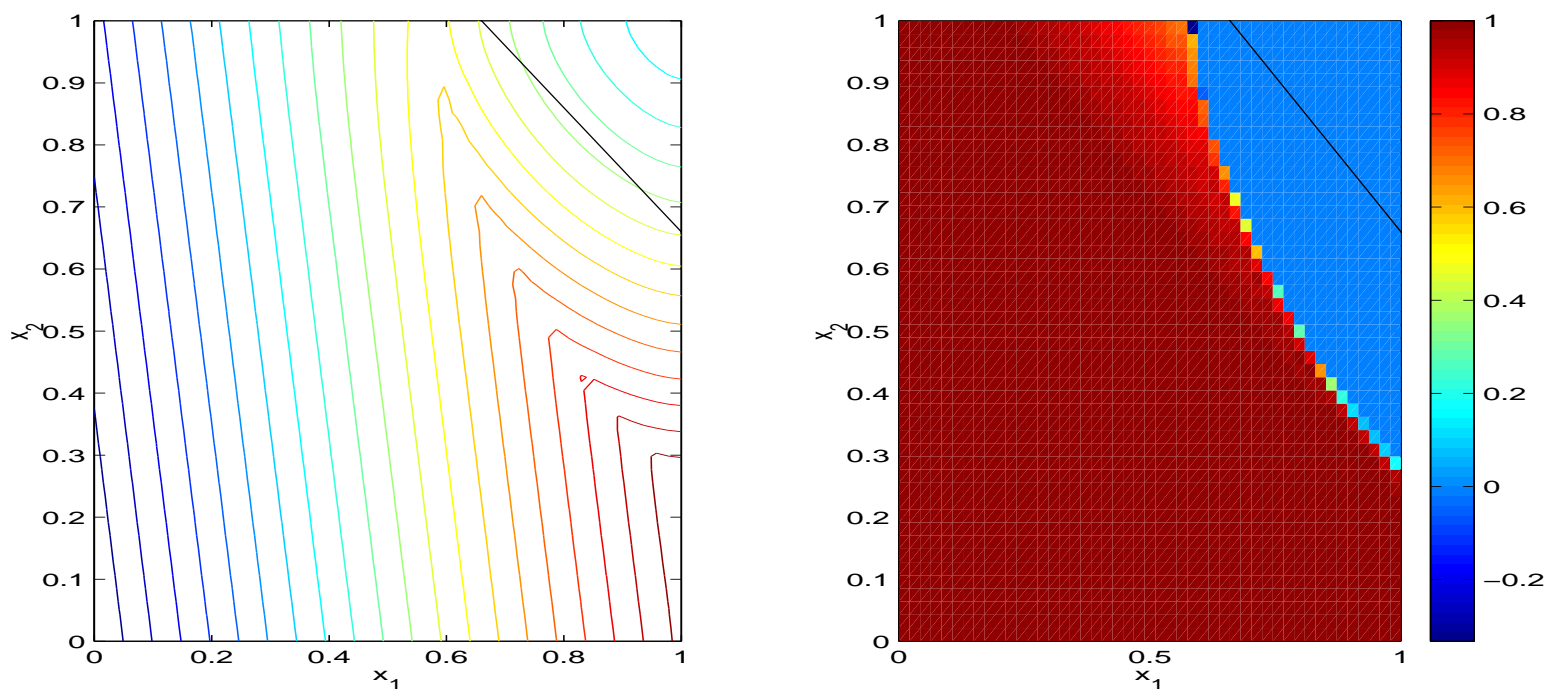

Figure 18: Level lines of the phase (left) and associated geometrical spreading $\beta$ (right) at time $2.8 \mathrm{~s}$. The dark line is the theoretical caustic. 


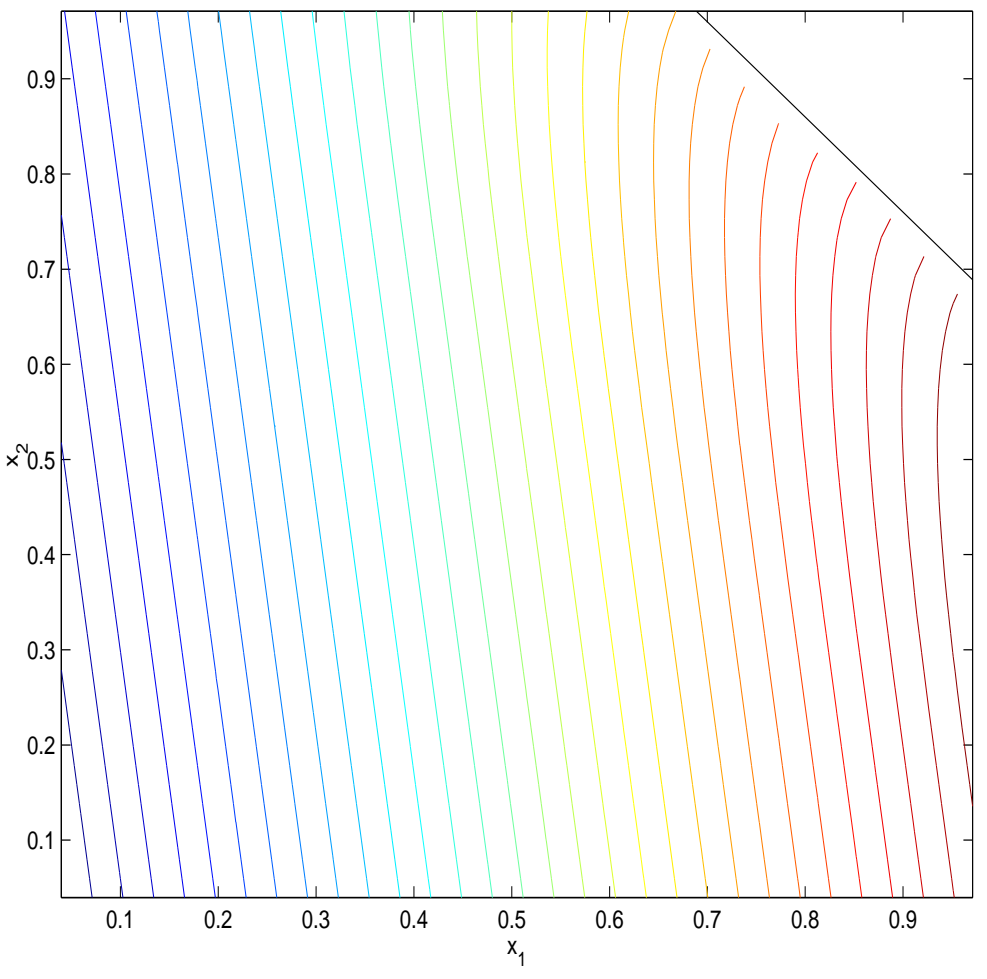

Figure 19: Level curves of the phase mapped back in the old time variable. Color map indicates the value of the phase. The dark line is the theoretical caustic. 


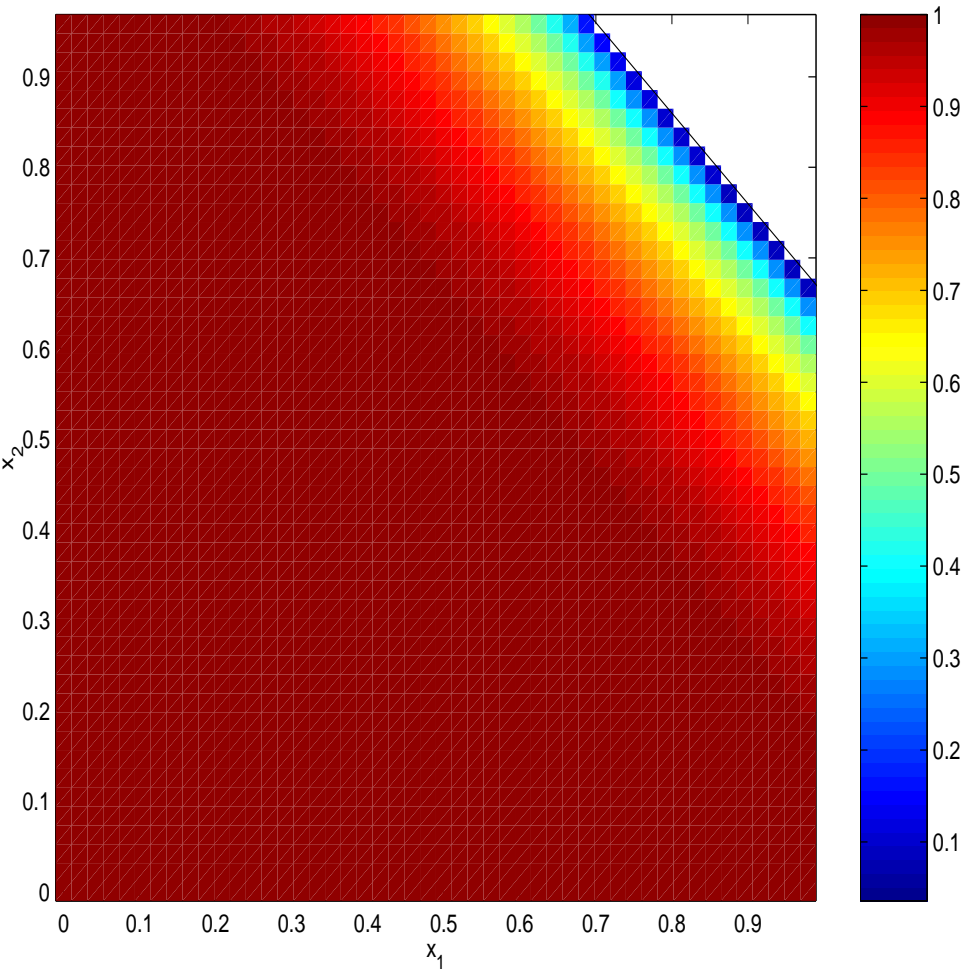

Figure 20: Eulerian geometrical spreading $\beta$ associated to the phase in figure 19. The white line is the theoretical caustic. 


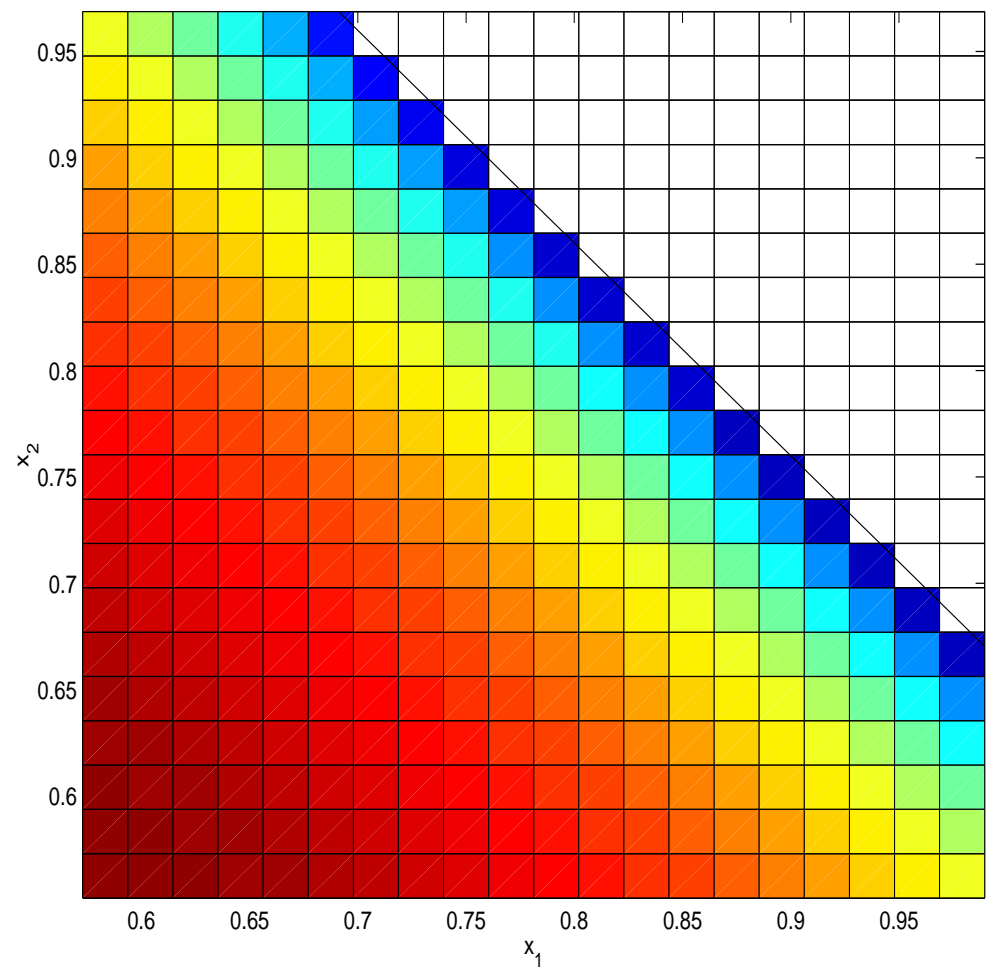

Figure 21: Zoom of figure (20) near the caustic.

Unit ${ }^{\prime}$ de recherche INRIA Lorraine, Technopôle de Nancy-Brabois, Campus scientifique, 615 rue du Jardin Botanique, BP 101, 54600 VILLERS LÈS NANCY

Unit'e de recherche INRIA Rennes, Irisa, Campus universitaire de Beaulieu, 35042 RENNES Cedex Unit'e de recherche INRIA Rhône-Alpes, 655, avenue de l'Europe, 38330 MONTBONNOT ST MARTIN Unit'e de recherche INRIA Rocquencourt, Domaine de Voluceau, Rocquencourt, BP 105, 78153 LE CHESNAY Cedex Unit ${ }^{\prime}$ de recherche INRIA Sophia-Antipolis, 2004 route des Lucioles, BP 93, 06902 SOPHIA-ANTIPOLIS Cedex 


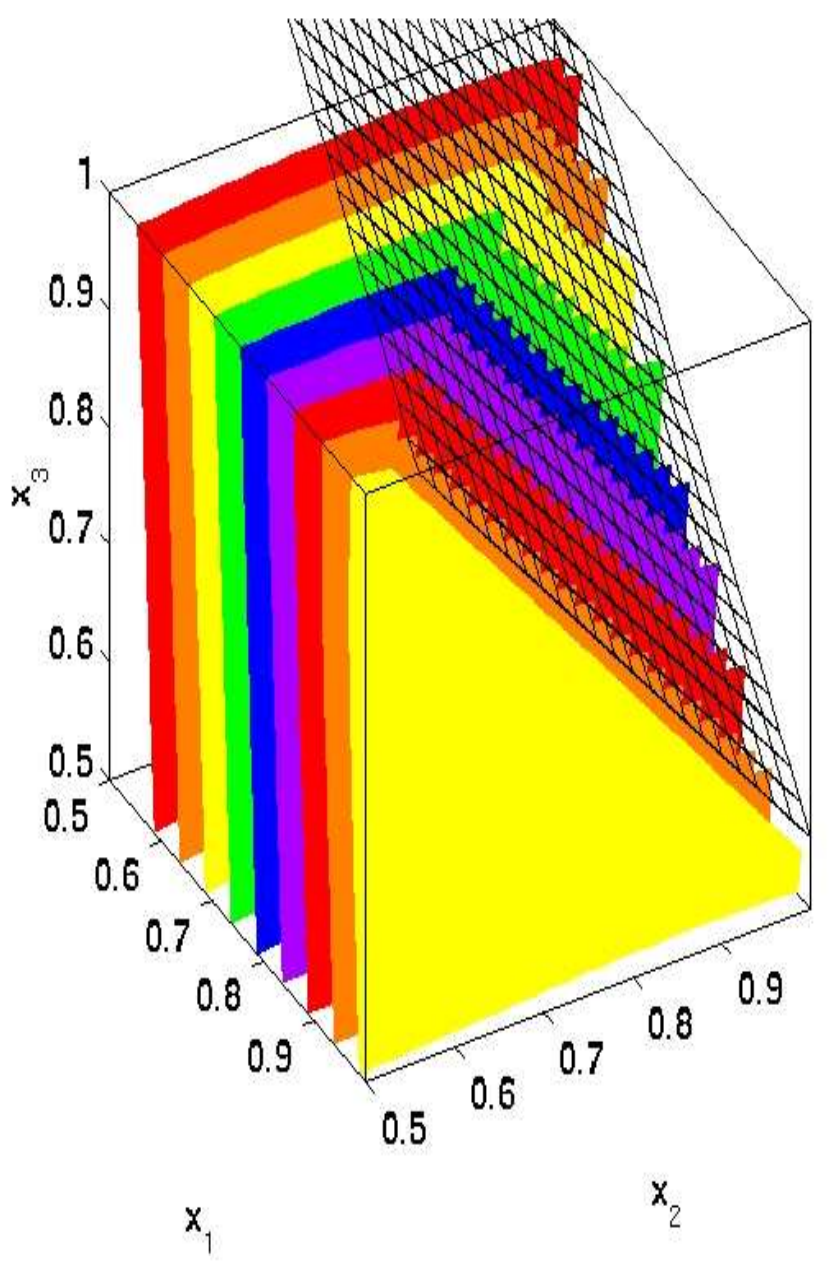

Figure 22: Case 1 : The isosurfaces of the phase (the colormap has now meaning) and the theoretical caustic surface as a black meshgrid. 


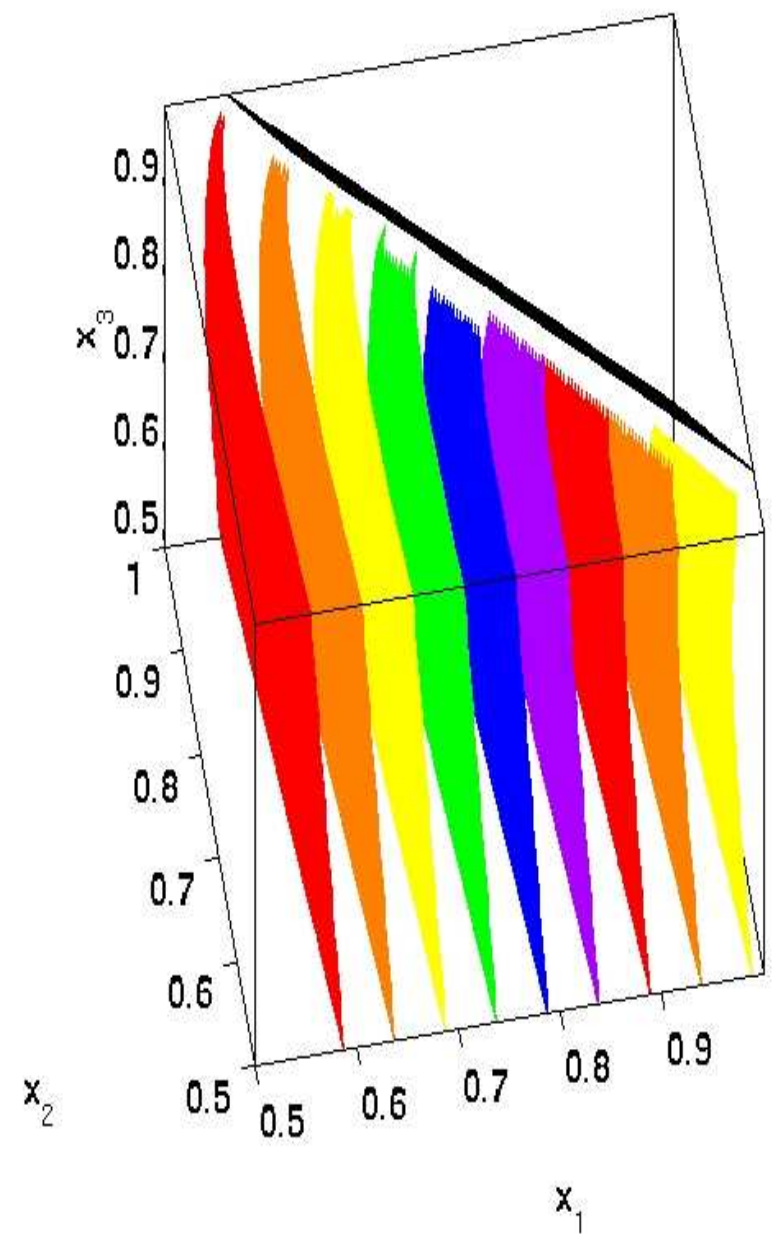

Figure 23: same as fig. 22 with different view point. 


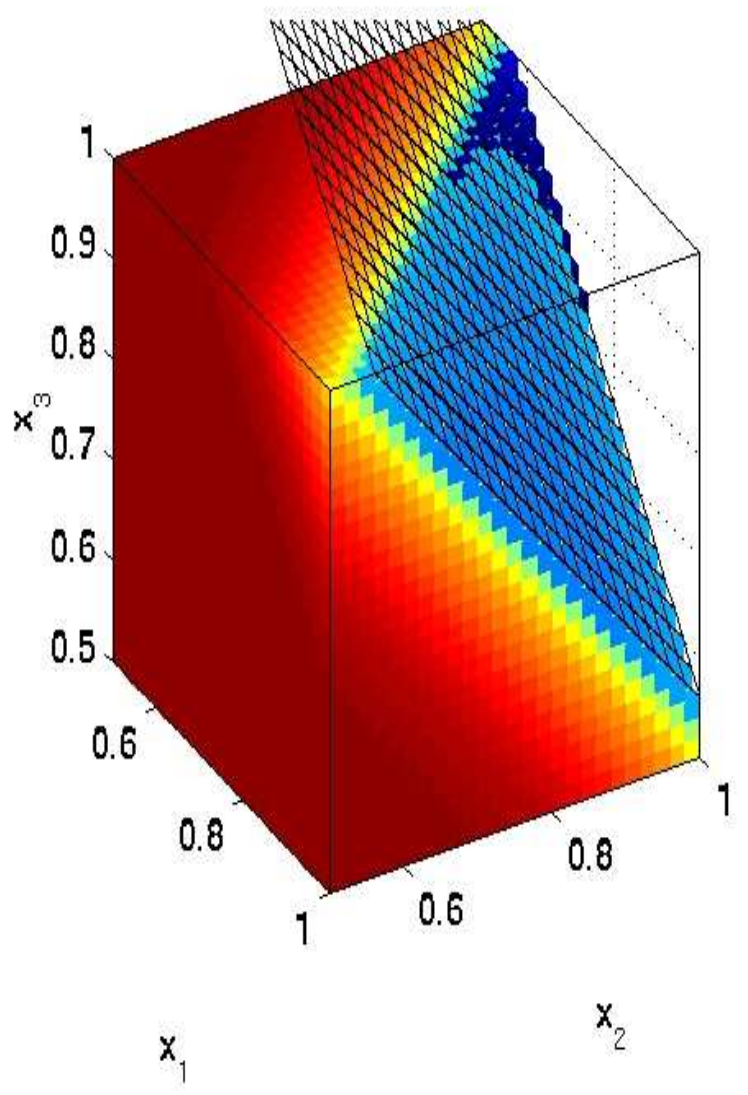

Figure 24: Case 1 : Eulerian geometrical spreading $\beta$ and the theoretical caustic surface as a black meshgrid. 


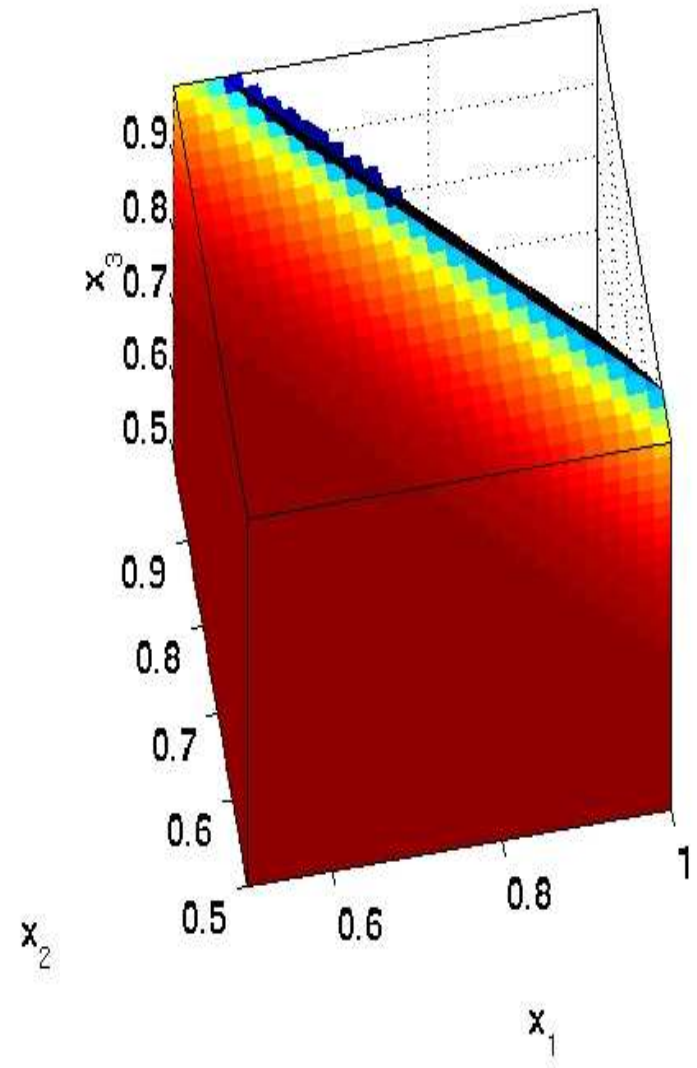

Figure 25: same as fig. 24 with different view point. 


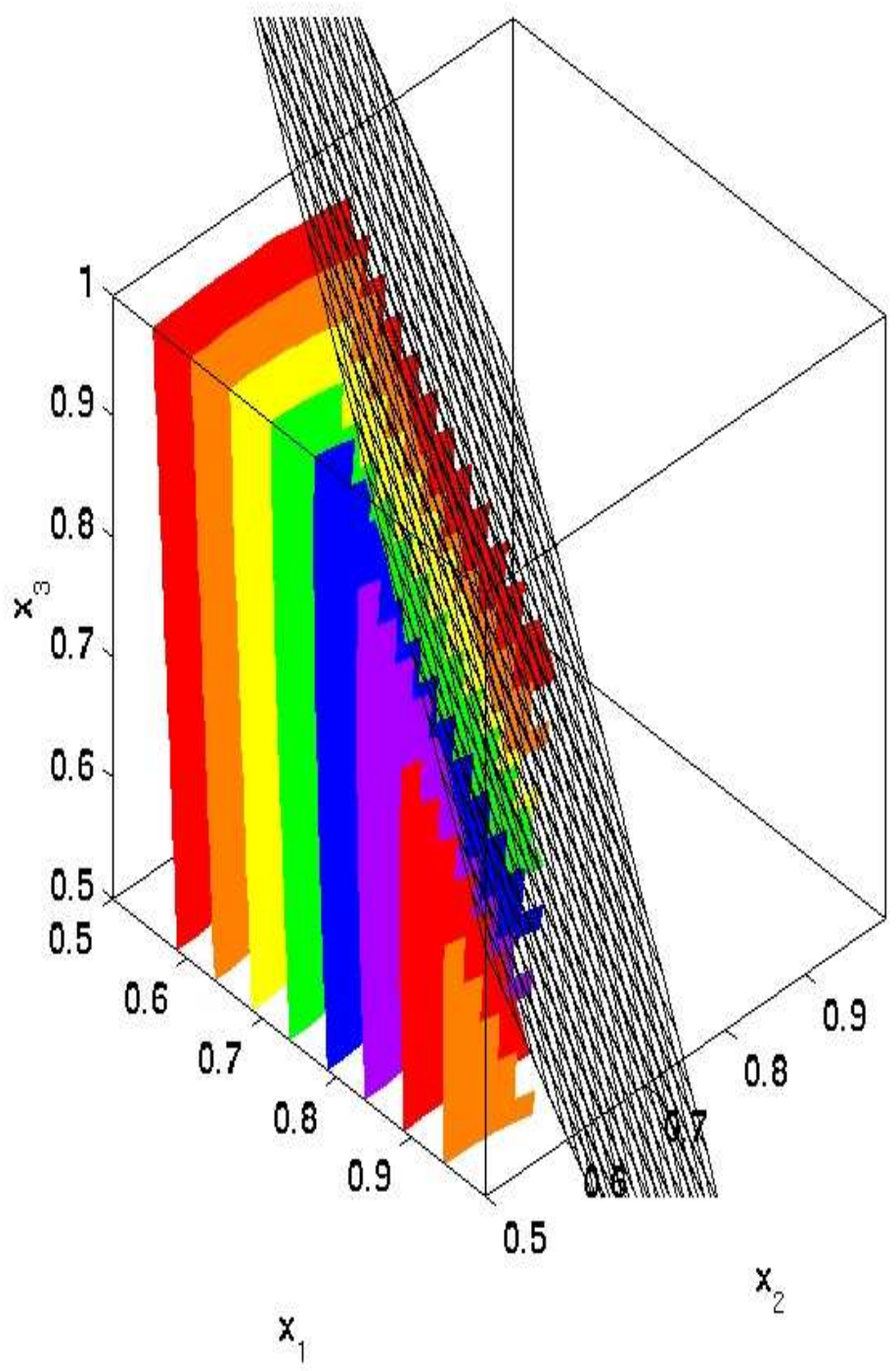

Figure 26: Case 2: The isosurfaces of the phase (the colormap has now meaning) and the theoretical caustic surface as a black meshgrid. 